Abstracts $^{*}$

\title{
48th National Congress of the Italian Society of Clinical Biochemistry and Clinical Molecular Biology (SIBioC - Laboratory Medicine)
}

\author{
Centro Congressi Lingotto, Turin (Italy) \\ 18-20 October 2016 \\ President of the Congress \\ Marcello Ciaccio (Palermo) \\ Scientific Committee \\ G. Lippi (Verona) - Coordinator
}

Fiamma Balboni (Firenze), Ettore Capoluongo (Roma), Ferruccio Ceriotti (Milano), Sergio Bernardini (Roma), Sabrina Buoro (Bergamo), Assunta Carnevale (Milano), Aldo Clerico (Pisa), Davide Farci Santarcangeli (Milano), Massimo Gion (Venezia), Maria Stella Graziani (Verona), Antonio La Gioia (Lari, PI), Silvia Mengozzi (Cesena), Gianpaolo Merlini (Pavia), Michele Mussap (Genova), Antonello Nonnato (Torino),

Roberta Pacifici (Roma), Paola Pezzati (Firenze), Massimo Quercioli (Firenze), Tommaso Trenti (Modena), Martina Zaninotto (Padova)

\section{Organising Committee}

Nino Cappuccia (Verbania), Maria Rita Cavallo (Pinerolo, TO), Giulio Mengozzi (Torino) Gianmatteo Micca (Asti), Marco Migliardi (Torino), Antonello Nonnato (Torino), Giovanna Patrucco (Torino)

\section{Scientific Secretariat}

G. Lippi (Verona) - Coordinator

Anna Lucia Caldini (Firenze), Ettore Capoluongo (Roma), Luca Deiana (Sassari), Maria Stella Graziani (Verona), Andrea Mosca (Milano), Antonello Nonnato (Torino)

Simona Pichini (Roma), Martina Zaninotto (Padova)

${ }^{*}$ These abstracts have been reproduced directly from the material supplied by the authors, without editorial alteration by the staff of this Journal. Insufficiencies of preparation, grammar, spelling, style, syntax, and usage are the authors. 


\title{
FROM HARMONIZATION TO THE BEST PRACTICE: EVIDENCE ABOUT POINT-OF-CARE TESTING
}

\author{
V. Pecoraro ${ }^{1}$, M. Mele ${ }^{2}$, T. Trenti ${ }^{1}$ \\ ${ }^{1}$ Laboratorio Tossicologia, Dipartimento interaziendale ad attività integrata Medicina di Laboratorio e Anatomia Patologica, Nuovo Ospedale \\ Civile Sant'Agostino Estense, Modena \\ ${ }^{2}$ Rete Aziendale PoCT, Dipartimento interaziendale ad attività integrata Medicina di Laboratorio e Anatomia Patologica, Nuovo Ospedale \\ Civile Sant'Agostino Estense, Modena
}

Background: Point of Care testing (PoCT) has been developed to provide rapid test results and improve patient management. PoCT for immunoassay are commonly utilized in emergency situations. Most published studies focus on analytical performance of PoCT, neglecting the importance of patients' outcomes ${ }^{1}$. We reviewed the analytical performance and diagnostic accuracy of PoCT specifically planned for immunoassay, comparing results obtained in PoCT and in standard laboratory settings, and we evaluated the possible impact of rapid results on patient management. Methods: We searched Medline and Embase for studies reporting immunoassay results obtained in both PoCT and central laboratory scenarios. We extracted data on study details and assessed the methodological quality of the studies. We evaluated the analytical characteristics and diagnostic accuracy of six PoCT: Tn, PCT, intraoperative PTH, BNP, CRP and N-Gal. Results: We analyzed 114 scientific papers. The majority of the studies (89,78\%) were judged at low risk of bias. Thirty five studies (31\%) reported analytical data, showing satisfactory correlations between results obtained through PoCT instruments and those in a central laboratory scenario. Fifty-four studies (47\%) evaluated the diagnostic accuracy of PoCT as acceptable for Tn (Sn 74\%,Sp 94\%), BNP (Sn 82\%,Sp 88\%) and CRP (Sn 85\%,Sp $85 \%$ ) only. Clinical impact was evaluated in 30 studies. Of these, 2 studies measuring PTH and BNP reported a limited impact on diagnostic decisions. Seven studies measuring CRP showed a significant reduction of antibiotic prescription. Several authors of Tn studies reported improved care through early diagnosis of ACS following PoCT. Conclusions: PoCT immunoassay results seem to be reliable and accurate in terms of diagnosis for Tn, BNP and CRP. The satisfactory analytical performance of PoCT, together with its excellent practicability, suggests that it could be a reliable tool in clinical practice, but, at present, no data shows a significant improvement in outcomes for patients treated in according to a PoCT protocol. The availability of quick test results seems to offers clinical advantages, but the impact of PoCT results is poorly documented in clinical studies.

1. Pecoraro V et al. Point-of-care testing: where is the evidence? A systematic survey. Clin Chem Lab Med 2014;52(3):313-24.

\section{THROMBOPOIESIS AND PLATELETS MORPHOLOGY}

\author{
G. Specchia \\ Bari
}

The history of identification of the platelets dates back to about 1800, when they were firstly defined as "dust of the blood", then in 1841 Addison called them "extremely minute ...granules and finally, in 1882, they were denominated "Platelets" by Bizzozzero. The term "Megakaryocytes" was coined by Howell in 1890. Since then, a series of experimental studies in vitro and in vivo has led to a knowledge of the complex molecular mechanisms that regulate both physiological and pathological Megakaryocytpoiesis/Thrombopoiesis. Megakaryocytopoiesis is the complex multistep process that takes place prevalently in the bone marrow, originating from the progenitor hemopoietic stem cells (HSCs) and ending with the production of polyploid Megakaryocytes that can release up to $10^{11}$ platelets (Thrombopoiesis) per day into the circulation to repair vascular damage and prevent hemorrhagic events. The proliferation/maturation process of Megakaryocytic Progenitors occurs through the biological mechanisms of endomitosis, producing cells with a polyploid nucleus and ample cytoplasm that express specific membrane antigens like CD41, CD42, CD61. The Thrombopoiesis process adds the crucial point in the phase when fragmentation of the pseudopodal projections of the mature megakaryocytes membrane gives rise to the proplatelets. This process involves a massive reorganization of the megakaryocytes membrane and cytoskeleton components, including actin and tubulin. The entire process of proliferation and differentiation of the Megakaryocytes is rigorously controlled and regulated by exogenous and endogenous factors as well as the transcriptional factors and epigenetic mechanisms. The prevalent extrinsic control mechanism of Megakaryocytopoiesis is carried out by Thrombopoietin (TPO) and its receptor MPL, expressed by the Megakaryocytes and their precursors. In turn, for signaling, they require the activity of the tyrosine kinase Janus 2 (JAK2). Thus, the TPO-MPL axis regulates both megakaryocytic maturation and platelets production. TPO acts in synergy with other cytokines like SCF, IL11 and EPO to promote the proliferation of HSCs. In addition, it has been demonstrated that after the activation of c-Mpl by binding with TPO, a series of intracellular molecular pathways is triggered, that involve JAK2 and then STAT3,STAT5, leading to the production of Bcl- $\mathrm{x}_{\mathrm{L}}$ SOCS, Cyclin D, p27 etc. Among the transcription factors that control megakaryocytopoiesis and thrombopoiesis, GATA1 and FLI1 play an important role. In addition, FLI1 acts in concert with other factors like GATA1-FOG1,ETS1 to activate the expression of many specific receptors of the megakaryocytes and platelets such as MPL, GP2B (CD41), GP9 (CD42). The discovery of the various molecular mechanisms that regulate the physiology of normal thrombopoiesis has contributed to a better knowledge of the pathogenesis underlying some hereditary/congenital platelets conditions as well as chronic myeloproliferative neoplasms. Today, light has been shed on the genomic defects involved in hereditary conditions that have long been known, and characterized by functional defects, such as the Bernard Soulier syndrome, "grey platelets" syndrome, Chediak-Higashi syndrome, Glanzman Thromboasthenia, etc. Hereditary 
thrombocytosis is rare, prevalently of dominant autosomal type, like those due to mutations of TPO, others to the MPL and JAK2 genes, but these are different from the acquired mutation V617F of JAK2 on exon 14 identified in 2005 in chronic myeloproliferative neoplasms (CMPNce). All diseases are characterized by specific morphological features of platelets.

\title{
PLATELETS: TECHNOLOGICAL ADVANCEMENTS TO MEET THE NEEDS OF CLINICAL MEDICINE
}

\author{
G. Da Rin \\ Laboratory Medicine, ASL 3 Bassano del Grappa, Italy
}

The assessment of the platelet count is essential in clinical hematology.

Historically, manual platelets count, using phase contrast microscopy, was common practice. The manual method is time-consuming and subjective.

The introduction of automated full blood counters resulted in a dramatic improvement in the accuracy and precision, particularly in severe thrombocytopenic patients.

The automated analytical procedures for platelet counting are: impedance analysis, optical light scatter/fluorescence analysis using various analysers and immunoplatelet counting by flow cytometry. (1) The impedance method detects cells by increase in electrical impedance when a cell passes through an aperture in the flow cytometer. Platelets are hereby detected by cell size because the increase in impedance is proportional to cell volume. For optical light scatter method, cells are also analysed based on volume when cells pass through a laser beam in the flow cytometer. If a two-angled method is used, light is two-dimensional permitting additional analysis of cell granularity. More accurate methods have been developed but are available on a few analysers. They include an immunological method with application of a plateletspecific antibody or fluorescent labeling of platelets prior to counting in the flow cytometer. Although currently in development, image analysis could become the standard practice in the future.

Other indices related to platelet counts are provided by hematologic analyzers.

They include mean platelet volume (MPV), platelet distribution width (PDW) and the fraction of large platelets. Derived platelet parameters are highly dependent upon the individual technology and are influenced by the anticoagulant and delay time from sampling to analysis.

The mean platelet component (MPC) based on the refractive index using an optical method corresponds to platelet density, and a decrease in density suggests platelet activation or defective biogenesis. Several clinical studies have documented an association between hemostasis and platelet size as they found MPV to be an independent risk factor for thrombosis, and large platelets are selectively consumed during massive bleeding. (2) No definite conclusion can be made about the value of any of the new platelet parameters to differentiate reactive thrombocytosis from clonal proliferation. (3) Reticulated platelets are newly released platelets; they contain RNA and are identified by flow cytometry and the use of nucleic acid specific dyes. Reticulated platelets are a laboratory tool to differentiate hypoproduction from accelerated platelet destruction and to predict platelet recovery after chemotherapy and stem cell transplantation $(2,3)$.

References

1. Briggs C, Harrison P, Machin SJ. Continuing developments with the automated platelet count. Int J Lab Hematol 2007;29(2):77-91.

2. Vinholt PJ, Hvas AM, Nybo M. An overview of platelet indices and methods for evaluating platelet function in thrombocytopenic patients. Eur J Haematol 2014;92(5):367-76.

3. Lecompte TP, Bernimoulin MP. Novel Parameters in Blood Cell Counters. Clin Lab Med 2015;35:209-224.

\section{DIFFERENTIAL DIAGNOSIS OF THROMBOCYTOPENIAS}

\author{
P. Noris, F. Melazzini \\ Department of Internal Medicine, IRCCS Policlinico San Matteo Foundation and University of Pavia, Pavia, Italy
}

The introduction into the routine laboratory practice of automated blood cell counters has much increased the prevalence of thrombocytopenia in the general population over the last decades. The main difficulty the clinicians must deal with is the correct recognition of inherited thrombocytopenias (ITs) from immune thrombocytopenia (ITP). This distinction can be difficult especially when a low platelet count is incidentally found in adulthood, and therefore categorized into acquired forms. The most important tools to prevent a misdiagnosis, and consequently inappropriate treatments, are careful medical history, physical examination, and microscopy examination of peripheral blood films (1). The presence of other family members with low platelet count, long lasting bleeding tendency even more pronounced than expected based on the degree of thrombocytopenia, and congenital or acquired additional defects, strongly supports the hypothesis of an IT. Nevertheless, recessive forms and de novo mutations must always be taken into account. Physical examination is important not only to detect the clinical signs of thrombocytopenias, as cutaneous or mucosal bleeding, but also to recognize the low platelet count as part of a syndromic IT: defects may affect all organs and apparatus, some of them are evident, while others must be sought carefully, eventually by imaging studies (1). Morphologic examination of peripheral blood smears is mandatory since it is the most informative tool for the differential diagnosis of thrombocytopenias: ITs are often characterized not only by morphological abnormalities of platelets, but also of leukocytes and/or red blood 
cells (2). Most of ITs are usually characterised by platelets of increased dimension, although the degree of this enlargement varies among the different inherited disorders. A classification of ITs based on platelet diameter has been suggested and it takes into account the percentage of platelets larger than $3.9 \mu \mathrm{m}$, which corresponds approximately to half the diameter of normal red blood cells. According to this classification, ITs are classified into four categories: with giant platelets, with large platelets, with normal or slightly increased platelet size, with normal or slightly decreased platelet size (3).

On the other hand, ITP is similarly characterized by increased platelet dimensions, largely overlapping those of ITs: a lower percentage of large platelets in ITP turned out to be a useful parameter for a correct differential diagnosis with IT with giant platelets $(3,4)$. Abnormality of platelet granules may also suggest the congenital origin of an isolated thrombocytopenia: in some ITs granules may be reduced or absent, more frequently associated with platelet macrocytosis. Polymorphonuclear leukocytes containing peculiar inclusion bodies (Döhle-like bodies) are specific for $M Y H 9$-related disease when associated with the presence of very large platelets, while some abnormalities of red cells morphology may suggest the diagnosis of rarer ITs. A few complex laboratory tests will substantiate the suspected diagnosis which is strongly recommended to be confirmed at molecular level given the predisposition to hematological cancer or to the appearance of additional defects worsening the quality of life, typical of some ITs (2).

References

1. Balduini CL, Pecci A, Noris P. Diagnosis and management of inherited thrombocytopenias. Semin Thromb Hemost 2013;39:161-171.

2. Balduini CL, Melazzini F, Pecci A. Inherited thrombocytopenias-recent advances in clinical and molecular aspects. Platelets 2016, Epub ahead of print. DOI:10.3109/09537104.2016.1171835.

3. Noris P, Biino G, Pecci A, et al. Platelet diameters in inherited thrombocytopenias: analysis of 376 patients with all known disorders. Blood 2014;124:e4-e10.

4. Noris P, Klersy C, Zecca M, et al. Platelet size distinguishes between inherited macrothrombocytopenias and immune thrombocytopenia. J Thromb Haemost 2009;7:2131-2136.

\title{
THROMBOCYTOSIS AND RISK OF THROMBOSIS IN MYELOPROLIFERATIVE NEOPLASMS
}

\author{
A. Falanga, V. Milesi, C. Giaccherini \\ Immunoematologia e Medicina Trasfusionale \& Centro Emostasi e Trombosi, Ospedale Papa Giovanni XXIII, Bergamo
}

Thrombotic events are very frequent and represent the main cause of morbidity and mortality in patients with Philadelphia chromosomenegative myeloproliferative neoplasms (MPNs), mainly Polycythemia Vera (PV) and Essential Thrombocythemia (ET) [1]. The pathogenesis of blood clotting activation in these diseases is multifactorial, and involves various abnormalities of platelets, erythrocytes, and leukocytes, as well as dysfunctions of endothelial cells. Typically, elevations in the number of circulating blood cells as well as modifications in the cellular hemostatic properties occur.

Patients with MPN can have a different thrombotic risk. Understanding this risk is very important as the treatment decision in these patients is highly dependent on their thrombotic risk category. According to age ( $>60)$, history of thrombosis, and, more recently, the presence or not of JAK2V617F mutation and/or cardiovascular risk factors: patients are stratified in "high-risk", "intermediate” or "low-risk" categories [2].

Thrombocytosis is typical of these diseases, particularly of ET, but its role in the pathogenesis of thrombotic events is still controversial. No studies to date have demonstrated a statistically significant correlation between the platelet count and thrombosis in either PV or ET. In the ECLAP study, neither the currently proposed therapeutic target of $400 \times 10^{9} / \mathrm{L}$ nor any other platelet count thresholds were able to predict a higher risk of thrombosis. In young ET patients (aged $<60$ years) with extreme thrombocytosis (platelet count $\geq 1,000 \times 10^{9} / \mathrm{L}$ ), and without a previous history of thrombo-hemorrhagic complications, the incidence of major thrombosis and hemorrhage during the follow-up was similar between those who were treated with prophylactic cytoreductive therapy and those who did not receive such therapy. These findings suggest that current treatment should not primarily aim at lowering the platelet count [3]. Paradoxically, extreme thrombocytosis (i.e., platelets $>1,500 \times 10^{9} / \mathrm{L}$ ) is rather associated with hemorrhagic manifestations in patients with ET. Recently, biological studies of circulating thrombotic markers, to characterize the presence of a hypercoagulable state in patients with MPN, show that platelet qualitative abnormalities, more than platelet count, are associated with hypercoagulability in these patients. Specifically, several studies have demonstrated that platelets circulate in an activated status and possess a high prothrombotic potential. These findings suggest that in these diseases the control of platelet activation over the platelet count is an important goal of treatment strategies.

Bibliografia

1. Barbui T, Finazzi G, Falanga A. Myeloproliferative neoplasms and thrombosis. Blood 2013;122(13):2176-84.

2. Tefferi A, Barbui T. Polycythemia vera and essential thrombocythemia: 2015 update on diagnosis, risk-stratification and management. Am J Hematol 2015;90(2):162-73.

3. Tefferi A, Gangat N, Wolanskyj AP. Management of extreme thrombocytosis in otherwise low-risk essential thrombocythemia; does number matter? Blood 2006;108(7):2493-4. 


\title{
CORRECTION WITHOUT PRE-WARMING OF COLD AGGLUTINATION IN THE RET OPTICAL CHANNEL OF SYSMEX XE-2100/XN 9000 AND MINDRAY BC 6800 HEMATOLOGICAL ANALYZERS. PRELIMINARY DATA
}

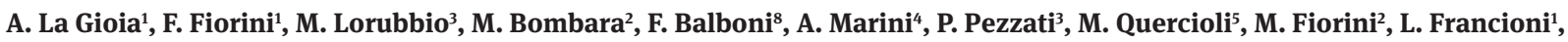 \\ E. Marchese $^{6}$, F. Maglione ${ }^{7}$ \\ ${ }^{1}$ Patologia Clinica Osp. Lotti, Pontedera \\ ${ }^{2}$ U.O.C. Medicina di Laboratorio Ospedale di Livorno \\ ${ }^{3}$ Laboratorio Generale - Azienda Ospedaliero Universitaria Careggi, Firenze \\ ${ }^{4}$ Patologia Clinica, Ospedale Versilia \\ ${ }^{5}$ Centro Regionale Controllo di Qualità AOU-Careggi, Firenze \\ ${ }^{6}$ Laboratorio Analisi I, AUO Policlinico-V. Emanuele, Catania \\ ${ }^{7}$ U.O.C. Patologia Clinica Venosa-Melfi, ASP - Potenza \\ ${ }^{8}$ Laboratorio Analisi Istituito Fiorentino di Cura e Assistenza, Firenze
}

Introduction. Cold agglutinin disease (CAD) is an autoimmune hemolytic anemia (AIHA) caused by an immune reaction against red blood cell self-antigens. The presence of agglutination on the EDTA tubes causes a decrease of RBC count and an increase of MCV. The MCHC increases in parallel with the artefactual decrease in the number of RBC. The warming at 37 degrees causes the reversibility of the agglutination and the normalization of the RBC counting as well as of the MCHC. It has been reported that the RBC value and the MCHC were not influenced by cold agglutination in the optical counting of RBC (RBC-O) in the RET channel of the Sysmex XE-2100 analyzers. In these cases, the RBC-O values are similar to the RBC counting performed after warming at 37 degrees in the impedance channel (RBC-I). We studied this phenomenon in a wider series of increased MCHC in order to evaluate the possibility to use the RBC-O counting instead of the RBC-I after warming. Methods. 35 samples with increased MCHC (range 375-2411g/L; median 540) were tested on the Sysmex XN 9000 and XE-2100 as well as on Mindray BC-6800 for Hemoglobin, RBC-O and for RBC-I after 2 hours of 37 degrees warming. MCHC in the optical channel was calculated by using a research parameter "Most Frequent Value" (MFV). Statistical analysis was performed using Analyse-it software version 3.90 .1 (Analyse-it software Ltd; Leeds, UK). Results. The RBC-O counting resulted in a resolution of the agglutination in 32 out of 35 samples. The comparison from two counting in 24 out of 27 samples analyzed with Sysmex technology showed a Passing Bablok regression y =,132 + 1,01 x $(95 \%$ CI of slope 0,87 -1,12 and intercept -0,304 to 0,635). The calculated MCHC-0 compared with the measured MCHC after 37 degrees warming showed a Passing Bablok regression $\mathrm{y}=-48,33+1,134 \mathrm{x}$ (95\% CI of slope 0,52-2,08 and intercept $-365,1$ to 153,0). Both RBC-O and MCHC -O resulted acceptable even by adding 8 Mindray samples to Sysmex samples. Conclusions. In $91,4 \%$ of the cases the RBC-O counting without pre warming resulted in a resolution of the agglutination. The MFV is proved a reliable surrogate of MCV to calculate the MCHC. For these reasons the Manufacturers should be encouraged to develop a reflex test for automatic execution of both RBC-O count and calculated indices in cases of the presence of cold agglutinins.

\section{BIOLOGICAL VARIATION OF PLATELET PARAMETERS DETERMINED BY THE SYSMEX XN-1000 HEMATOLOGY ANALYZER}

\author{
M. Seghezzi, B. Manenti, P. Dominoni, A. Crippa, L. Cerutti, S. Buoro \\ SMeL Generale di Base - Analisi Chimico Cliniche Azienda Socio Sanitaria Territoriale Papa Giovanni XXIII
}

Aim of the study: Biological variation data (BVD) allow the derivation of important parameters for the interpretation and use of laboratory test, including indices of individuality (II), assessment of significance of change in serial results (reference change values, RCV). As BVD can be impacted by analytical technology, we evaluated BVD of hematology parameters provided by the Sysmex XN1000. In this study we present the results for PLT parameters (PLT count; PDW, Platelet Distribution Width; MPV, Mean Plaletet Volume; P-LCR, Platelet Large Cell Ratio; PCT, platecrit). Material and methods: Blood samples were collected by the same phlebotomist at weekly intervals over 5 weeks from 21 ostensibly healthy volunteers (12 women, 9 men with ages ranging from 26 to 64 years old) and analyzed in duplicate within 2 hours using a single Sysmex XN analyzer. The analytical (CVa), within-subject (CVi) and between-subject (CVg) component of variations were calculated by nested ANOVA from replicated analyses after Cochran's test and Reed's criterion for outlier identification. Shapiro-Wilk test was applied separately to the results of each individual to check data distribution. Results: CVa ranged from 1.0\% (PLT) to 3.1\% (PDW); CVi and CVg were 6.4 and 15.4 for PLT, ranging from 2.3 (MPV) to 7.0 (P-LCR) and from 7.3 (MPV) to 21.2 (P-LCR) respectively; Index of Individuality (II) was lower than 0.5 for all platelet parameters. RCV was 18.1 for PLT, 7.3 for MPV, 14.5 for PDW, 21.1 for P-LCR, 17.8 for PCT. Conclusions: As shown by the results of CVa, the analytic variation of Sysmex XN for PLT parameters are very low, particularly for PLT count (CVa $=1.0 \%)$. CVi and CVg were compatible or slightly lower in comparison with published data on desiderable quality specifications. The II was $<0.5$ for all PLT parameters, indicating high individuality and limited diagnostic utility of reference intervals in the detection of unusual results in an individual. Therefore, comparison of serial results to detect significant changes should be evaluated by means of RCV. The improvement of precision of new analytical technologies implies greater confidence in the use and documentation of BVD, that is an essential prerequisite mainly in development of clinical application of new parameters. 


\title{
CSF ANALYSIS IN ACUTE MENINGOENCEPHALITIS: BIOCHEMICAL AND CYTOLOGICAL APPROACH
}

\author{
G. Bernardi, E. Corsini, M. Lazzaroni \\ Clinical Pathology and Medical Genetics Laboratory, Fondazione IRCCS INN C. Besta, Milano
}

Biochemical and Cytological analysis offer initial etiological diagnosis of acute meningoencephalitis, while microbiological and viral results confirm diagnose, identify causative agent and optimize treatment. Due to high morbidity and mortality, rapid diagnosis for prompt and appropriate treatment is necessary. Each hospital laboratory has to ensure urgently CSF glucose, Total Protein (TP) and cytometry. In bacterial meningitis glucose consumption differentiate them from viral ones, CSF/serum glucose ratio gives better sensitivity and specificity, but serum is not always available in emergency. TP rise much in bacterial meningitis and in a lower degree in viral meningitis, international and national guidelines $(1,2)$ suggest to prefer to TP CSF/serum Albumin quotient, but in emergency total protein is largely preferred. Cell count and their differentiation is essential for diagnosis, reference method is microscopy and counting chamber, but cell type differentiation needs expert operators. Modern Blood cells analyzers have now specific CSF counting programs, each one has different performances that are well described in literature (2). As meningoencephalitis involves high number of cells, sensitivity is not a priority. Most attention has to be made in cell differention as granulocytes are typical in bacterial meningitis, lymphocytes in viral one and monocytes in resolution phases. A lot of new biomarker have been recently proposed, possibility to test them in emergency is an important selection criteria. There are a lot of publications on CSF C Reactive Protein, IL6 and Procalcitonin. Antigens from various agents are tested in CSF to anticipate cultural results, Galactomannan antigen in CSF has been recently inserted in diagnostic criteria (3). Problems arise from interpretation results as intrathecal fraction is usually not detected and absolute values are influenced by barrier function and serum values that may be very high in case of invasive pathogen disease. Viral meningoencephalitis have usually a benign clinical course, but sometimes can worsen, so lumbar puncture is made in post-acute phase when viral PCR test are negative, unfortunately CSF serology is rarely performed according to CSF standards $(1,2)$, mainly because serology and biochemistry are made in different laboratories. So while there is perfect harmonization in routine CSF analysis, other tests need greater collaboration among the various laboratory specialties.

1. Deisenhammer F, Bartos A, Egg R, et al. Guidelines on routine cerebrospinal fluid analysis. Report from an EFSN task force European Journal of Neurology 2006;13:913-922.

2. Bernardi G, Brunati P, Biagioli T, et al. L'analisi del liquido cefalorachidiano. Cerebrospinal fluid (CSF) analysis. Biochim Clin 2014;38;238-254.

3. Chong GM, Maertens JA, Lagrou K, et al. Diagnostic Performance of Galactomannan Antigen Testing in Cerebrospinal Fluid. J Clin Microbiol 2016;54:428-431.

\section{BACTERIAL MENINGITIS}

\author{
S. Andreoni \\ Novara
}

Meningitis is an infection of the protective membranes covering the brain and spinal cord. Acute bacterial meningitis is the most common form of meningitis. Approximately 80 percent of all cases are acute bacterial meningitis. Bacterial meningitis can be life threatening.

The bacteria most often responsible for bacterial meningitis are common in the environment and can also be found in your nose and respiratory system without causing any harm. Sometimes meningitis occurs for no known reason. Other times it occurs after a head injury or after you have had an infection and your immune system is weakened. Outbreaks of bacterial meningitis also occur in living situations where you are in close contact with others, such as college dormitories or military barracks.

Community-acquired bacterial meningitis is still a significant cause of morbidity and mortality. The organisms that cause bacterial meningitis differ somewhat by geographic region and by age. In premature babies and newborns up to three months old, common causes are group B streptococci and bacteria that normally inhabit the digestive tract such as Escherichia coli. Older children are more commonly affected by Neisseria meningitidis (meningococcus) and Streptococcus pneumoniae and those under five by Haemophilus influenzae type B. In adults, Neisseria meningitidis and Streptococcus pneumoniae together cause $80 \%$ of bacterial meningitis cases. Risk of infection with Listeria monocytogenes is increased in persons over 50 years old. The introduction of pneumococcal vaccine has lowered rates of pneumococcal meningitis in both children and adults

The epidemiologic features of bacterial meningitis have changed dramatically over the past decades with the advent of the Haemophilus influenzae vaccine.

The most common symptoms are fever, headache and neck stiffness. Other symptoms include confusion, vomiting, and an inability to tolerate light or loud noises. Young children often exhibit only nonspecific symptoms, such as irritability, drowsiness, or poor feeding.

To diagnosis bacterial meningitis, CSF examination is mandatory. CSF culture is the "gold standard" for diagnosis, and it is obligatory to obtain the in vitro susceptibility of the causative microorganism and to rationalize treatment. CSF Gram staining, latex agglutination testing are additional diagnostic tools that might aid in etiological diagnoses, especially for patients with negative CSF cultures. If lumbar puncture cannot be performed, serum inflammatory marker, blood culture, skin biopsy, and urine antigen testing may provide supportive evidence to diagnose bacterial meningitis. 
More specific technologies are under development to enable more confident diagnosis of the major etiologic agents of meningitis. One example of a technology that could potentially be applied broadly to meningitis is that of a PCR multiplex panel.

Bacterial meningitis is treated with antibiotics. A general intravenous antibiotic with a corticosteroid to bring down the inflammation may be prescribed even before all the test results are in. In addition to antibiotics, it will be important to replenish fluids lost from loss of appetite, sweating, vomiting.

\section{References}

1. Bahr NC, Boulware DR. Methods of rapid diagnosis for the etiology of meningitis in adults. Biomark Med 2014;8:1085-1103.

2. Brouwer MC, Tunkel AR, van de Beek D. Epidemiology, Diagnosis, and Antimicrobial Treatment of Acute Bacterial Meningitis. Clinical Microbiology Reviews 2010;23:467-492.

3. Ku LC, Boggess KA, Cohen-Wolkowiez M. Bacterial meningitis in the infant. Clin Perinatol 2015;42:29-45.

\section{MENINGITIS WITH A CLEAR CEREBROSPINAL FLUID}

\section{Giraldi \\ Cosenza}

Introduction: The infections of the central nervous system are very heterogeneous due to the broad spectrum of microorganisms (bacteria, viruses, fungi, helminths) that cause them, and on account of the clinical aspect. Based on the clinical syndromes they can be classified into: acute meningitis, subacute and chronic, acute and chronic encephalitis, space-occupying lesion syndrome, myelitis, neuritis, radiculitis, (the latter in particular are often mediated by bacterial toxins).

Meningitis/encephalitis: They are inflammation of the meninges, usually of infectious origin, which may evolve, if not properly treated, in meningo-encephalitis or brain-myelitis.

Acute meningitis in adults is characterized by high fever, headache gravativa, exacerbated by contacts, movements, lights, sounds, cerebral vomiting, and stiff neck. Viral meningitis is characterized by the onset of acute meningeal symptoms and fever and can affect all ages, but it is most common in young children. The main ethiologic agents are enteroviruses, responsible for $46 \%$ of viral meningitis, followed by herpes simplex -2 (HSV-2) (31\%), varicella zoster (VZV) (11\%) and HSV-1 (4\%). Following the introduction of the vaccine, the incidence of viral meningitis due to mumps and measles has been reduced, while meningitis caused by enteroviruses, has slightly increased especially those caused by Coxsachie B and Echovirus. Enterovirus meningitis may be accompanied by mucocutaneous manifestations including vesicles localized on the hands, feet and mouth, herpangina and generalized maculopapular rash. HSV also causes viral meningitis as a complication of primary genital infection (especially HSV-2) and is not always associated with clinical symptoms. Meningitis due to VZV is most commonly observed in association with the reactivation of the virus and can occur in $50 \%$ of cases in the absence of skin lesions. Even the primary HIV infection is an important cause of meningitis whose neurological symptoms can occur in $17 \%$ of cases of seroconversion. Viral meningitis agents involved in forms of the traveler and that affect humans almost exclusively in the warm seasons, a period in which arthropod vector activities are present and they are: West Nile virus (WNV), St. Louis encephalitis virus, Tick- viruses borne encephalitis (TBE) Virus and Tuscany (TOSV).

Encephalitis is an inflammation of the brain tissue supported by an infectious cause, viral, and less frequently, bacterial or parasitic. The diagnosis etiology is only possible in half of the cases.

The symptoms are: agitation, altered state of consciousness and possible seizures. In nursing infants usually it prevails the seizure symptoms. The encephalitis can be primitive or represent the secondary complication of a viral infection or a vaccination. The most frequent forms of primary encephalitis are due to HSV-1, HSV-2, VZV, enterovirus and influenza virus A. The HSV encephalitis is the most frequent, with a 'incidence of about 1/million per year. It can be the consequence of a primary infection or a reactivation and is caused in $90 \%$ of cases by HSV-1 and 10\% in HSV-2. The HIV patients, with immunosuppression, may develop encephalitis mainly by: HSV, VZV and cytomegalovirus. The arbovirus encephalitis, transmitted by mosquitoes and ticks (fever of St. Louis, Eastern and Western equine fever, fever of California, neuroinvasive forms of WNV, tick-borne encephalitis “tick-borne encephalitis"), affect humans almost exclusively in the warm seasons, a period in which the activity of the arthropod vector is present.

Laboratory diagnosis of meningitis or encephalitis: The cerebrospinal fluid (CSF) undergoes microbiological, physical, chemical and biochemical examination. Blood cultures are also performed as support together with other microbiological tests of various biological samples not belonging to the neurological district. Serum can also be tested.

Collection and transport of CSF sample and other samples: LCR: is taken by lumbar puncture (LP) or lumbar puncture in the space between the 4 th and the 5 th lumbar vertebra, collected in sterile tubes with screw cap and conical bottom. The sequential division into three aliquots help gradually reduce contamination with blood from the tissue perforated during the execution of the lumbar puncture. The sample should be collected, preferably before beginning antimicrobial therapy and sent to the laboratory within 15-30 minutes of collection and no later than a maximum of two hours along with blood samples for a possible serological diagnosis, swabs (nasal, pharyngeal and vesicular), feces for the eventual search of enteroviruses and blood cultures.

Examination of the CSF: The examination of the CSF includes: macroscopic and microscopic evaluation, chemical-physical investigations, direct research of bacterial and fungal antigens, culture and susceptibility and specific gene amplification test.

In viral meningoencephalitis the CSF has an increased protein content, cell count with a predominance of peripheral blood mononuclear cells which are between $>5<500 \mathrm{~GB} / \mathrm{mL}$ (lymphocytosis may be absent at the beginning of the infection). The glucose levels are usually normal 
or low in the course of mumps virus infection, HSV, enteroviruses, HIV, WNV, and LCMV. Microscopic examination can reveal red blood cells which may be present especially in encephalitis caused by HSV. In viral meningitis (a clear CSF) specific gene amplification tests for each virus are diagnostic.

Bibliography

- Meyding-Lamadé U, Strank C. Herpesvirus infections of the central nervous system in immunocompromised patients. Ther Adv Neurol Disord 2012;5(5):279-96.

- UK Standards for Microbiology Investigations, Investigation of Cerebrospinal Fluid Bacteriology, 10.07.2012 B 27, Issue no: 5.1

- Shin SY, Kwon KC, Park JW, Kim JM, Shin SY, Koo SH. Evaluation of the Sleeplex Meningitis ACE Detection Kit for the Detection of 12 Common Bactreial and Viral Pathogens of Acute Meningitis. Ann Lab Med 2012;32:44-49.

- Fabbrini G, Berardelli A. Meningite e altre malattie del sistema nervosa. La neurologia della Sapienza, 2012.

- Favaro M, Savini V, Favalli C, Fontana. A multi-target real-time PCR assay for rapid identification of meningitis-associated microorganisms. C Mol Biotechnol 2013;53(1):74-9.

- Prasad, Rajniti, et al. Serum procalcitonin in septic meningitis. The Indian Journal of Pediatrics, 2013 - Journal of Evolution of Medical and Dental Sciences 2013;2:18.

- Sherkatolabbasieh, Zare, Kansestani,Shafiezadeh, Mahruei. Serum Procalcitonin level and other biological markers in children with bacterial or non-bacterial meningitis. Journal of Paramedical Sciences 2013.

- Stahl JP. Meningiti acute EMC - Neurologia 2013;13:2.

- Studahl M, Lindquist L, Eriksson BM, Günther G, Bengner M, Franzen-Röhl E, Fohlman J, Bergström T, Aurelius E. Acute viral infections of the central nervous system in immunocompetent adults: diagnosis and management. Drugs 2013;73(2):131-58.

- Bellini R, Calzolari M, Mattivi A, et al. The experience of West Nile virus integrated surveillance system in the Emilia-Romagna region: five years of implementation, Italy, 2009 to 2013. Euro Surveill 2014;19(44). pii: 20953.

- Circolare Ministeriale per la Sorveglianza dei casi umani di Chikungunya, Dengue, West Nile Disease ed altre arbovirosi e valutazione del rischio di trasmissione in Italia, 2015.

\title{
THE CRUCIAL ROLE OF CLINICAL LABORATORY: TOSCANA VIRUS AND LISTERIA EXPERIENCES
}

\author{
P. Pauri \\ UOC Patologia Clinica, Jesi - AV2- ASUR Marche; Study Group SIBioC “Infectious disease diagnostics”
}

The diagnosis of acute meningo-encephalitis in the emerging setting is very important for many reasons: to begin the specific therapy as soon as possible, for prognostic evaluation, to identify and limit epidemic disease, to plan public sanitary approaches for control and prevention and to improve the local epidemiologic knowledge.

The synergic actions between Clinical Laboratories and Public Health Services are more relevant in cases of meningitis and meningo-encephalitis caused by Toscana Virus and Listeria which are zoonotic infections.

The project founded by the Italian National Center for Disease Prevention and Control (CCM) called "Surveillance of the zoonoses and the vectorial diseases: rationalization of the diagnostics methods and of information flows for the planning of interventions in the medical and veterinarian fields" started in 2014 in Marche, Emilia-Romagna, Lombardy and Sicily. The project is based on the evidence that the laboratory plays a strategic role in the clinical diagnostic process as well in the notification of zoonoses to the competent health authorities.

In 2016, in the Italian Society of Clinical Biochemistry and Laboratory Medicine (SIBioC), the "Infectious Disease Diagnostics Study Group" was born. Its initial task is a survey to evaluate the "state of art" in the Italian laboratories, of diagnostic serology comprehensive of zoonoses. The Toscana Virus (TOSV) belongs to the Bunyaviridae family and it is the most frequent pathogen for the aseptic meningitis with a good prognosis. This pathogen is spread by Phlebotomus perniciosus and Phlebotomus perfliewi, also known as vectors for human and canine leishmaniosis. For this reason, the TOSV meningitis is widespread in summer time both in the center of Italy (30-52\% of the cases) and in the Mediterranean basin $(1,2,3)$. The laboratory diagnosis must be considered in all those cases of meningitis with clear liquor and lymphocytosis that occur from June to October.

Gene amplification as well as IgG and IgM antibodies research with recombinant antigens are first choice tests. This approach allows us to avoid useless tests and to reduce the hospitalization. As a consequence, surveillance programs and disinfestation campaigns can be started.

Listeria monocytogenes (LM) rarely causes sepsis and hard meningitis. It is a food pathogen which causes an high number of hospitalization with a mortality rate from $20 \%$ to $30 \%$. In the ministerial protocol (2015), Listeriosis is classified as an invasive bacterial disease (4) that is mandatory to notify. Since 2015, there is in Marche Region an epidemic outbreak which has involved 23 people with 3 deaths. The infection, clinically characterized by invasive and systemic forms, is caused by the same genotype (cluster) that was identified by using the pulsed field gel electrophoresis (PFGE) and next generation sequencing (NGS).

According to CCM projects, Clinical Laboratories, Clinicians, Prevention Departments and Veterinarians have operated in a coordinated and integrated way. Also Public Health Services as well as the Zoo-prophylactic Institutes of Marche and Umbria, Abruzzo and Molise Regions were involved. 
Bibliography

1. Charrel RN, Bichaud L, Xavier de Lamballerie A. Emergence of Toscana virus in the mediterranean area. World J Virol 2012;1(5):135-141.

2. Valassina M, Meacci F, Valensin PE, Cusi MG. Detection of neurotropic viruses circulating in Tuscany: the incisive role of Toscana virus. J Med Virol 2000;60:86-90.

3. Pierro A, Landini MP, Gaibani PA, et al. A model of laboratory surveillance for neuro-arbovirosis applied during 2012 in the Emilia-Romagna region, Italy. Clin Microbiol Infect 2014;20(7):672-7.

4. Protocollo per la sorveglianza nazionale delle malattie batteriche invasive da meningococco, pneumococco ed emofilo e delle meningiti batteriche in Italia (versione del 24/7/2015).

5. Petruzzelli A, Blasi G, Masini L, et al. Occurrence of Listeria monocytogenes in salami manifactured in the Marche Region (Central Italy). J Vet Med Sci 2010;72:499-502.

\title{
THERAPEUTIC DRUG MONITORING OF ANTI-TNF BIOLOGIC AGENTS: WHAT BENEFIT FOR PATIENTS?
}

\author{
V. Pecoraro, E. De Santis, T. Trenti \\ Laboratorio Tossicologia, Dipartimento interaziendale ad attività integrata “Medicina di Laboratorio e Anatomia Patologica”, Nuovo \\ Ospedale Civile Sant’Agostino Estense, Modena
}

\begin{abstract}
Background: Infliximab and adalimumab are biologic blockers anti-inflammatory drugs directed against TNF $\alpha$, that reduce the inflammatory response of autoimmune diseases. Biologics are immunogenic, and consequent anti-drug antibodies (ADA) are usually determined for therapeutic drug monitoring to optimize dosing of anti-TNF $\alpha$ (1). We performed a systematic review to evaluate the impact of anti-drug antibodies (ADA) on therapeutic response.

Methods: We considered studies enrolling adult patients affected by Rheumatoid Arthritis (RA), Ulcerative Colitis (UC) and Psoriatic Arthritis (PsA) in therapy with infliximab or adalimumab. We searched electronic databases MEDLINE and Embase. We collect data about study and population characteristics, treatment dosage, determination of ADA, adverse events and reaction to site of infusion. We combined data in meta-analysis, calculating risk ratios (RR) for each study. We evaluated heterogeneity calculating $\mathrm{I}^{2}$. P-values $<0.05$ were considered as statistically significant. Analyses were performed with the RevMan 5.3 and Stata 11 softwares.

Results: We included 16 studies enrolling 2289 patients. Of these, 2166 patients (95\%), evaluated in 13 studies, were affected by RA, 85 by UC ( 1 study) and 38 by PsA ( 2 studies). DAS28 score at baseline ranged from 5.2 to 6.5. The effect of ADA on treatment response was evaluated in 8 studies, showing a significant reduction of response (RR 0.75, 95\%CI $0.6-0.94$ ) in patients with ADA+ respect to patients ADA-. The heterogeneity between studies was low $\left(\mathrm{I}^{2}=6.3 \%\right)$. The therapy with infliximab or adalimumab produced, reaction of infusion site in 73 patients ( $\left.3 \%\right)$, infection in 168 (7\%) patients and serious adverse events in less of $1 \%$ of patients (20 out of 2289).

Conclusion: Our analysis showed that detectable ADA reduced anti-TNF $\alpha$ response. ADA could interfere with drugs and compromise their effects. Moreover, they cause injection site reaction and infections. The development of ADA and consequent worse therapeutic response is well documented (2,3), but the usefulness of determination of ADA remains unclear (4). At moment, there are any indications about use of immunogenicity test to guide therapy. We need of more information to define the usefulness of this test for patients management.
\end{abstract}

\section{Reference}

1. Vande Casteele N, Gils A. Pharmacokinetics of Anti-TNF monoclonal antibodies in inflammatory bowel disease: adding value to current practice. J Clin Pharmacol 2015;55(Suppl 3):S39-50.

2. Atzeni F, Talotta R, Salaffi F, Cassinotti A, Varisco V, Battellino M, Ardizzone S, Pace F, Sarzi-Puttini P. Immunogenicity and autoimmunity during anti-TNF therapy. Autoimmun Rev 2013;12(7):703-8.

3. Moss AC, Brinks V, Carpenter JF. Review article: immunogenicity of anti-TNF biologics in IBD - the role of patient, product and prescriber factors. Aliment Pharmacol Ther 2013;38(10):1188-1197.

4. Mok CC, Tsai WC, Chen DY, Wei JC. Immunogenicity of anti-TNF biologic agents in the treatment of rheumatoid arthritis. Expert Opin Biol Ther 2016;16(2):201-11.

\section{CONTROL AND VALUE OF LABORATORYIN THE DIAGNOSTIC APPROPRIATENESS}

\author{
C. Bonaguri' ${ }^{1}$ A. Melegari² \\ ${ }^{1}$ Diagnostic Laboratory Department, Parma Hospital, Parma, Italy \\ ${ }^{2}$ Diagnostic Laboratory Department, NOCSAE Hospital, Modena, Italy
}

Background. The limitation of inappropriate test requests and the identification of a balance between available economic resources and increasing health needs is crucial for modern Healthcare Services. In this perspective, harmonization of testing algorithms is a goal. To improve the appropriateness of the test requests in autoantibody testing, reliable and universally accepted diagnostic algorithms need to be 
defined and implemented; these algorithms should have been developed using the available guidelines found in the current scientific literature and should be shared by all physicians working in clinical immunology. The most appropriate strategy for requesting autoimmune rheumatic disease laboratory testing should encompass selective criteria, should begin from a clinical suspicion, followed by a logical succession of analyses performed with sensitive tests at an early stage and specific tests for confirmation. We have specifically focused on antinuclear antibodies (ANA), Extractable Nuclear Antigens (ENA) and double-stand (dsDNA).

The Emilia-Romagna Region, on March 1th, 2013 has released the first coding for ANA Reflex testing, a common guideline for autoantibody testing in Autoimmune Rheumatic Disease, which places ANA test at the first analytical level whereas the following steps are directly guided by the laboratory. With this algorithm, we can also specify the presence/absence of anti-mitochondria antibodies (AMA) . The natural history of $\mathrm{PBC}$ may be significantly improved when the disease is diagnosed at an early stage. Anti-mitochondria antibodies (AMA) are the serological mainstay for the diagnosis of PBC at present, but they cannot be detected in a significant percentage (i.e., 10-15\%) of PBC patients, thus resulting in a delayed diagnosis. Some antinuclear antibodies (ANA),such as anti-Multiple nuclear dots and anti-Membranous/Rime-like which target the sp100 and gp210 antigens respectively, are regarded as PBC-specific surrogate biomarkers,although controversial data exist about their diagnostic and prognostic value.

Methods. We have compared both the number of ANA, ENA and dsDNA tests and the percentage of positive results at the second level tests observed in the first three years after the implementation of ANA Reflex (May-Dicember 2013, 2014, 2015) in Autoimmune Laboratories of Parma and Modena.

Results. During the years after ANA Reflex implementation we have observed that the requests of ANA Reflex showed a significant increasing trend of total ANA requests in both Parma (45\%, 54\%, 70\%) and Modena (54\%, 61\%, 80\%). We have also found that ENA and dsDNA requests during this period showed a reduction in Parma and in Modena, accompanied by a substantial increase of positivity, with an improvement in selected positive cases.

Conclusions. Close collaboration and audit between clinicians and laboratory personnel will enable standardization and widespread implementation of diagnostic algorithms for a more efficient use of immunological tests in the diagnostic evaluation, prognostic assessment, and monitoring of patients with systemic autoimmune diseases.

References

1. Shoenfeld Y, Cervera R, Haass M, et al. EASI - The European Autoimmunity Standardisation Initiative: a new initiative that can contribute to agreed diagnostic models of diagnosing autoimmune disorders throughout Europe. Ann N Y Acad Sci 2007;1109:138-44.

2. Conrad K, Roggenbuck D, Reinhold D, et al. Autoantibody diagnostics in clinical practice. Autoimmun Rev 2011 May 18 [Epub ahead of print].

3. Bonaguri C, Melegari A, Ballabio A, et al. Italian multicentre study of a diagnostic algorithm in autoantibody testing for autoimmune rheumatic: conclusive results. Autoimmun Rev 2011;11:1-5.

\title{
THE NEW TEST FOR MONITORING ANTI-TNF $\alpha$ THERAPY: FROM LABORATORY TO THE CLINICAL PRACTICE
}

\author{
E. De Santis ${ }^{1}$, A. Melegari' ${ }^{1}$, C. Bonaguri' ${ }^{2}$, T. Trenti ${ }^{1}$ \\ ${ }^{1}$ Diagnostic Laboratory Department, Nocase of Modena \\ 2Diagnostic Laboratory Department, Parma Hospital
}

Background: Biologicals directed against TNF $\alpha$ have improved the treatment of autoimmune disease. However, there are patients who do not respond or show a loss of efficacy becouse of the formation of anti-drug antibodies (ADA). Aim: Evaluating the test for individual monitoring of therapy, the relevance of ADA detection and the relationship with clinical response and some laboratory parameters. Materials and methods: We enrolled 73 patients treated with Infliximab (IFX): 12 with Ulcerative Colitis and 25 with Crohn's disease from Gastroenterology Unit of Parma, 6 with Rhetumatoid Arthritis, 6 with Psoriatic Arthritis and 24 with Ankylosing Spondylitis from Rheumatolgy Unit of Modena. Blood samples were collected before the next IFX somministration and we tested: IFX drug level, ADA and some aoutoantibodies characteristic of each disease. Results: From Gastroenterology: 18,92\% of patients show IFX in therapeutic range without ADA, 24,33 IFX in subtherapeutic level without ADA, 13,51\% IFX in therapeutic range with ADA, 43,24\% with IFX in subtherapeutic level with ADA. From Rheumatolgy: $21,4 \%$ of patients show IFX in therapeutic range without ADA, 16,7\% IFX in subtherapeutic level without ADA, 45,2\% IFX in subtherapeutic level with ADA and 16,7\% FX in therapeutic range with ADA. The correlation with clinical response suggest that the presence of ADA could interfere with efficacy of therpay but there is no significant association beetwen ADA and other serological parameters. Conclusion: These tests for monitoring therapy are easy and quick but with a high cost. The detection of ADA and IFX levels is important tools in order to prevent adverse events and therapy failure but we have to optimize this approach trought collaboration between clinical and laboratory.

Reference

Atzeni F, et al. Immunogenicity and autoimmunity during anti-TNF therapy. Autoimmun Rev 2013;12(7):703-8. 


\title{
NEXT GENERATION SEQUENCING (NGS) APPLICATIONS IN THE STUDY OF METABOLIC LIPID DISEASES
}

\author{
A. Di Costanzo \\ Department of Internal Medicine and Medical Specialties, “La Sapienza” University, Policlinico Umberto I Hospital, Rome, Italy
}

In an age when the majority of monogenic human disease genes have been identified, one of the challenges for the incoming generation of human geneticists will be to unravel the mechanism of polygenic and multifactorial diseases such as primary or secondary disorders of lipid metabolism, metabolic syndrome (MetS), Type 2 diabetes (DT2), hepatic steatosis (NAFLD) which lead to cardiovascular disease (CVD).

These complex phenotypes are caused by a multitude of genetic and environmental factors acting in concert, which involves the inheritance and expression of a phenotype being determined by many genes at different loci, with each gene exerting a small additive effect. This implies that the different effects are cumulative, i.e. no one gene is dominant or recessive among another.

Genome-wide association studies (GWAs) have revealed several loci and several common single nucleotide polymorphisms -SNP- (Minor Allele Frequencies, MAF $\geq 5 \%$ ) associated with multifactorial metabolic diseases and plasma lipid levels. However, the proportion of risk of lipid phenotypes explained by GWAS-identified loci remain modest [1].

It has been suggested that rare (MAF $\leq 1 \%$ ) or low-frequency variants (MAF $=1 \%-5 \%)$ with moderate/strong effects that are not captured by GWAS, could explain a part of the 'missing heritability' [2-3]. An effective way to identify these rare/low-frequency variants is to re-sequence the candidate genes or the whole-genome in subjects with extreme phenotypes or perform a case-control studies. This strategy has already been successfully employed for various candidate genes involved in lipid metabolism, where multiple rare variants in combination with common variants were found to contribute to inter-individual variation in plasma lipid levels [4-5].

Mutational analysis of many genes, however, requires massive parallel sequencing technologies (NGS) to be accomplished reliably and within a reasonable time. In the last few years, the advent of NGS technologies has revolutionized the approach to genetic studies, making wholegenome sequencing a possible way of obtaining global genomic information. NGS has been shown to be successful in identifying novel causative mutations of rare or common Mendelian disorders. The identification of rare and frequent genetic variants can be very important in clinical practice to detect pathogenic mutations or to establish a profile of risk for the development of pathology. The purpose of this presentation is to discuss the recent applications of NGS in the study of several multifactorial metabolic lipid diseases, also discussing some relevant studies conducted to evaluate the genetic variance of plasma lipid-lipoprotein traits. We will also illustrate some preliminary data from our experience in NGS technologies used to identify genetic markers of NAFLD disease.

1. Teslovich TM, Musunuru K, Smith AV, et al. Biological, clinical and population relevance of 95 loci for blood lipids. Nature 2010;466:707-713. 7 Willer CJ, Schmidt EM, Sengupta S et al. Discovery and re finement

2. Manolio TA, Collins FS, Cox NJ, et al. Finding the missing heritability of complex diseases. Nature 2009;461:747-753. 9.

3. Marian AJ, Belmont J. Strategic approaches to unraveling genetic causes of cardiovascular diseases. Circ Res 2011;108:1252-1269.

4. C Cohen JC, Kiss RS, Pertsemlidis A, Marcel YL, McPherson R, Hobbs HH. Multiple rare alleles contribute to low plasma levels of HDL cholesterol. Science 2004;305:869-872.

5. Razzaghi H, Aston CE, Hamman RF, Kamboh MI. Genetic screening of the lipoprotein lipase gene for mutations associated with high triglyceride/low HDL-cholesterol levels. Hum Genet 2000;107:257.

\section{LABORATORY DIAGNOSTICS OF DYSLIPIDEMIAS: HARMONIZATION OF RESULTS AND REPORTING}

\author{
F. Ceriotti \\ Clinical Laboratory Service, San Raffaele Hospital, Milano
}

Dyslipidemias represent in western countries a relevant modifiable cardiovascular risk factor. Lipid measurements are the tool to identify the presence of dyslipidemias and to monitor their treatment. The existence of agreed upon decision limits (even if different in Europe and USA) requires a high degree of trueness and precision of the measurements of all the lipid components. For this reason it is extremely important to control all the sources of pre-analytical and analytical variability. Pre-analytical. The patient has to be in a stable metabolic state, without any inflammatory disease (that reduces TC) and the blood drawing has to be performed after a sitting position of at least 5 min and with a short tourniquet time to avoid possible hemoconcentration. Fasting is preferable even if a recent consensus statement indicates that it is not required in most cases. Analytical. Due to the fact that the decision limits for all lipids are not on the tails of the distribution of the population values, but in the middle, even a small bias introduces a large misclassification of subjects, so the use of methods able to provide traceable results is mandatory. For total cholesterol (TC), LDL-Cholesterol (LDL-C), HDL-Cholesterol (HDL-C) and Triglycerides (TG) a reference measurement system exists and it is maintained by the Centers for Disease Control and Prevention (CDC) (Atlanta, GA) that coordinate a network of reference laboratories. For TC and TG the reference measurement system is based on a pure primary standard (cholesterol and triolein) and ID- GC-MS reference methods (for TC also the Abell-Kendall method), for HDL-C and LDL-C pure cholesterol remains the primary standard, but the results depend on the CDC method used for the separation of the class of lipoproteins. 
Producing accurate lipid results is important, but without a proper reporting the outcome for the patient may be null or even negative. And reporting the lipids results is particularly complex. A recent survey in Italy has shown an incredible heterogeneity. In fact the use of population based reference intervals is not recommended because of the unhealthy lipid concentrations typical of the western populations. On the contrary it is suggested to use decision limits based on desirable lipid concentrations, necessary to reduce the cardiovascular risk. Unfortunately the desirable lipid concentrations depend on the patient's characteristics, some of these characteristics (smoke, hypertension, diabetes, previous cardiovascular events, etc.) are not known by the laboratory. To avoid the making of complex reports with several possible references, it has been decided to propose a uniform simplified reporting indicating the desirable values for a patient at intermediate - low level of cardiovascular risk (targets: TC $\leq 5.00 \mathrm{mmol} / \mathrm{L}, \mathrm{LDL}-\mathrm{C} \leq 3.00 \mathrm{mmol} / \mathrm{L}, \mathrm{HDL}-\mathrm{C} \geq 1.00 \mathrm{mmol} / \mathrm{L}$ (males), $\geq 1.20 \mathrm{mmol} / \mathrm{L}$ females, TG $\leq 1.70$ $\mathrm{mmol} / \mathrm{L}$ ). These references have to be accompanied by a comment indicating that, in case of subjects with high cardiovascular risk, lower targets should be considered. Only combining correct analytical results and proper reporting, the service for the patient will be really effective.

\title{
GENETIC SCREENING OF FAMILIAL HYPERCHOLESTEROLEMIA: KEY ROLE IN CARDIOVASCULAR PREVENTION
}

\author{
C. Giacobbe', M.D. Di Taranto ${ }^{2}$, A. Morrone², G. Marotta ${ }^{3}$, M. Gentile ${ }^{3}$, P. Rubba ${ }^{3}$, G. Fortunato ${ }^{2}$ \\ ${ }^{1}$ CEINGE S.C.ar.l. Advanced Biotechnology, Naples \\ ${ }^{2}$ Department of Molecular Medicine and Medical Biotechnology, University of Naples Federico II, Naples \\ ${ }^{3}$ Department of Clinical Medicine and Surgery, University of Naples Federico II, Naples
}

Introduction. Familial Hypercholesterolemia (FH) is a common autosomal dominant disorder characterized by high levels of total and LDL cholesterol, associated with increased cardiovascular risk (1). Early identification of FH patients can be useful to establish an adequate therapy and prevent cardiovascular accidents. We aim to screen the main genes involved in FH. Patients and Methods. We enrolled 466 subjects with clinically diagnosed FH, of whom 342 were unrelated.The promoter and exon 18 of the LDLR gene, exons 26 and 29 of the APOB gene as well as all exons of the PCSK9 and LDLRAP1 genes were amplified by PCR and directly sequenced. MLPA was performed to identify large rearrangements in the LDLR gene. Results. The screening of candidate genes revealed mutations in 265/342 unrelated FH patients, of whom 239 have mutation in LDLR gene, 4 in PCSK9 gene, 2 in APOB gene and 1 in LDLRAP1 gene; 12 patients are homozygotes or compound heterozygotes. We also observed that the values of LDL cholesterol gradually incresead among the homozygous or compound heterozygous patients, heterozygous patients with a null mutation (splicing, nonsense, duplication and deletion), heterozygous patients with missense mutations and patients without mutations, with statistically significant difference ( $\mathrm{p}=0.001)$. Conclusions. The screening of the LDLR, APOB, PCSK9 and LDLRAP1 genes revealed a mutation rate equal to $77.5 \%$. Carriers of radical mutations showed a severe lipid phenotype suggesting a strict follow up and an early initiation of therapy. In conclusion, molecular diagnosis is an effective instrument to early detect affected patients with a view to improving the prevention of fatal cardiovascular events.

1. Do R, et al. Exome sequencing identifies rare LDLR and APOA5 alleles conferring risk for myocardial infarction. Nature 2015;518(7537):102-6.

\section{CLINICAL APPLICATION FOR THE STUDY OF THE CHOLESTEROL METABOLISM BY MASS SPECTROMETRY}

\author{
V. Leoni ${ }^{1}$, A. Nauti ${ }^{1}$, C. Caccia ${ }^{2}$ \\ ${ }^{1}$ Laboratorio Analisi Chimico-cliniche, Ospedale di Circolo di Varese, ASST-Settelaghi, Varese \\ ${ }^{2}$ Laboratorio di Patologia Clinica e Genetica Medica, IRCCS Carlo Besta, Milano
}

Cholesterol is a structural elements of the plasma membrane. About half of the circulating cholesterol is absorbed and half is synthesized from acetyl-CoA. High serum cholesterol is related to higher cardiovascular risk. Evidences of a metabolic impairment were described in several neurodegenerative diseases (NDs), as Alzheimer, Huntington and Multiple Sclerosis. Oxysterols (-OHC) are mono-oxygenated cholesterol metabolites formed by autoxidation (7-ketocholesterol, 7KC) or by the activity of specific enzymes expressed in tissues as liver, brain and lung. Methods. Sterol and oxysterol profiling in serum (or plasma) is measured by isotope dilution mass spectrometry after alkaline hydrolysis and liquid to liquid extraction. Results. Plasma lathosterol, lanosterol and desmosterol (precursor sterols) are markers of the cholesterol biosynthesis. They increase in hypercholesterolemia and decrease in the statin therapy. They are markedly increased in some rare autosomal recessive metabolic diseases and reduced in NDs. In presence of inhibition of the citric acid cycle and OXPHOS, the lower formation of ATP and NADHH significantly reduces the cell and tissue levels of sterol precursors. Plasmatic 24OHC (formed in brain by neuronal CYP46A1) depends on the number of metabolically active neurons in the cerebral cortex and decreases in proportion to the degree of brain atrophy in several NDs. Plasma 270HC (formed by CYP27A1) increased significantly in hypercholesterolemia and more than 100\% in SPG5, an autosomal recessive disorder characterized by peripheral neurodegeneration. In the presence of atherosclerosis with carotid thickening, the concentration of 7KC (and 270HC) is increased. 7KC is involved in apoptotic mechanisms observed in ischemia-reperfusion and demyelination 
process. Conclusions: The sterol and oxysterol profile is a diagnostic test for rare inherited metabolic diseases and provides information about the whole cholesterol metabolism. The evaluation of the clinical and diagnostic significance of this metabolomic information in aging and degenerative diseases is in progress: new pathogenetic mechanisms, new biomarkers and new therapeutic targets have been identified so far.

\title{
FREQUENCY OF BRCA1 AND BRCA2 LARGE GENOMIC REARRANGEMENTS (LGRS) IN MUTATION- NEGATIVE OVARIAN CANCER WOMEN CANDIDATES TO OLAPARIB TREATMENT
}

\author{
R. Rizza, A. Costella, G.L. Scaglione, D. Guarino, C. Santonocito, A. Minucci, P. Concolino, E. Capoluongo \\ Laboratory of Molecular Biology, Institute of Biochemistry and Clinical Biochemistry, Catholic University of Sacred Heart, Policlinico "A. \\ Gemelli”, Roma
}

Background: Ovarian cancer is the leading cause of death from gynecological malignancies. Olaparib is an oral PARP inhibitor, used as postchemotherapy maintenance in high-grade serous ovarian cancer (HGSC) patients with germline or somatic mutations in BRCA1 or BRCA2. However, emerging data may be able to help expand its role into tumors with other homologous recombination deficits (while also determining if all BRCA1/BRCA2 mutations respond equally). Large genomic rearrangements (LGRs) have recently been identified in hereditary breast and/or ovarian cancer (HBOC) families and account for a small but still significant proportion of cases in several populations. 81 BRCA1 and 17 BRCA2 variants large genomic rearrangements have actually been described and are responsible for up to one-third of the identifiable BRCA mutations. Patients and Methods: Fifty-seven patients with HGSC, were fully screened for BRCA1/2 mutations by massive parallel sequencing. All samples negative were tested for BRCA1 and BRCA2 rearrangements using the Multiplex Amplicon Quantification (MAQ), following the manufacturer's protocol. Amplification products were analyzed with 3500 - Genetic Analyzer (Applied Biosystems Warrington, UK) and results were analyzed by the software MAQ-S v2.0 (Multiplicom) for quantification of copy number variation (CNV). Results: The MAQ method has detected 11 LGRs in BRCA1 gene, divided as follows: 4 deletions in exons 1-2, 2 macrodeletions from exon1 to exon 13, 1 deletion in exon 3, 1 deletion in exon 14 and 1 in exon 17. Between the rearrangements detected, we identified two novel rearrangements: exon 3 duplication and exon 1-6 deletion in BRCA1 gene. Conclusion: The treatment of recurrent ovarian cancer remains a big challenge to clinicians. Clinical and molecular characterization of LGRs identified in HGSC patients is necessary for therapeutic management. For this reason, we recommend the use of specific approach, as MAQ technique, after massive parallel sequencing in order to ensure an improvement of quality of NGS pipeline.

\section{THE CLINICAL VALUE OF PHARMACOTOXICOLOGY LABORATORY REPORTS IN DETERMINING THE “USE/NON-USE” OF DRUGS}

\author{
C. Leonardi \\ Direttore Dipartimento Dipendenze e Medicina Penitenziaria, ASL Roma 2
}

According to the Italian occupational health legislation, every worker has to be checked by an occupational specialist in function of his occupational risk.

Distress occurs when repeated or excessive stimuli exceed the adaptive capacity of the individual, resulting in manifestation of a pathological condition that can involve various systems like the cardiovascular, nervous, endocrine, gastrointestinal and the immune systems. Any heavy or stressful work conditions are fertile ground for the establishment of the psychopathological situation and/or may contribute to cause a chronic stress-related illness.

Bus drivers of public transport are the workers with higher levels of stress. The traffic of the city, relationships with passengers, the responsibility for people transporting, stressful shifts often in the conditions of isolation, the inability to decide when have a work break, shift work and repetitive tasks are critical stress factors for these workers.

Cocaine is the best stimulant drug and it is one of the most addictive drugs in the world. Hundreds of thousands of individuals are victims of the nervous effects caused by cocaine. These effects may be different in every person. Since it is one of the top stimulants, it is able to cause some very powerful effects and for these reasons it is still one of the most abused drugs in the world.

While the use of cocaine in the workplace is quite common, heroin is less used among workers.

Cocaine may successfully mask fatigue; however, high dosages may to impair judgment and to interfere with the ability of the driver to concentrate. Coordination and vision are impaired. There is an increase in impulsive behaviours with tendencies to take more risks and create confusion within the user. A person using cocaine maintains the illusion of being alert and stimulated, although physical reactions are impaired.

Also our statistics show that more transportation workers are using illegal drugs, with a large spike in cocaine use.

Cocaine harms both employees and employers, and despite the downward trend, it is still a serious issue. It is a very addictive drug, and when used at work, it can have disastrous effects.

Worker's distress may be a cause for this prevalent use and abuse of cocaine. 
References

1. Strand MC, Gjerde H, Mørland J. Driving under the influence of non-alcohol drugs - An update. Part II: Experimental studies. Forensic Sci Rev 2016;28(2):79-101.

2. Zhao J, Macdonald S, Borges G, Joordens C, Stockwell T, Ye Y. The rate ratio of injury and aggressive incident for alcohol alone, cocaine alone and simultaneous use before the event: a case-crossover study. Accid Anal Prev 2015 Feb;75:137-43.

3. MacDonald S, Mann R, Chipman M, Pakula B, Erickson P, Hathaway A, Mac Intyre P. Driving behavior under the influence of cannabis or cocaine. Traffic Inj Prev 2008;9(3):190-4.

\title{
NEW PSYCHOACTIVE SUBSTANCES AND NEW BIOLOGICAL MATRICES
}

\author{
S. Pichini, E. Marchei, M.C. Rotolo, M. Pellegrini, S. Graziano, R. Pacifici \\ Istituto Superiore di Sanità, Roma
}

\begin{abstract}
New psychoactive substances (NPS) are increasingly emerging on illegal drug market. In 2015 Europol and European Monitoring Centre for Drugs and Drug Addiction reported the identfication of more than 150 new substances mainly sold by internet web sizes and whose effects are naively reported by usres in web forums. Many cases of co-consumption of NPS and other substances have also been reported. Hence, the development of analytical methods aiming at the detection of a broad-spectrum of compounds (NPS and "traditional" drugs) are needed in case of seizures of unknown products or intoxiaction by unknown substances.

We will provide a critical overview of the analytical methods for detection and quantification of NPS and traditional substances in non biological (seizures) and biological matrices.

Systematic toxicological analysis in case of completely unknown samples will be also illustrated.

Focus will lie on advances in sample preparation, analytical techniques and new biological matrices for clinical and forensic purposes. Liquid chromatography-tandem mass spectrometry (LC-MS/MS) makes it possible to determine low concentrations not only in serum, plasma or whole blood or urine, but also in alternative matrices like oral fluid, dried blood spots, hair, nails and non biological matrices. Ultra-high performance liquid chromatography-tandem mass spectrometry (UHPLC-MS/MS), recently introduced in our analytical laboratory makes it possible to quantify a high number of compounds within a shorter run time with a minimum use of solvents and simple matrices extraction.

Analytical methods using these techniques can identify new synthetic cannabinoids, new synthetic cathinones, phenetilamines-derived substances, triptamines, etc..

Systematic toxicological analysis is currently applied to conventional clinical and forensic matrices but also to new biological matrices used to disclose NPS use, such blood dried spots which represent a future challenge to non invasively investigate on NPS related intoxications.
\end{abstract}

\section{NEW PREGABALIN ASSAY: POSSIBLE ABUSE}

A. Calcinari, M. Brugia, A.M. Margarucci Gambini, M. Piaggesi, S. Marinelli, M. Galeazzi

Laboratorio Analisi, Azienda Ospedaliero Universitaria Ospedali Riuniti, Ancona

Pregabalin (PRG) is a gamma-aminobutyric acid (GABA) analogue indicated for the treatment of neuropathic pain, add-on therapy for partial seizures in epilepsy and generalized anxiety disorder, benzodiazepine addiction.We aimed to evaluate the possible abuse liability of PRG among patients from drug addiction service (Ser.T.) and ER with suspect of benzodiazepines abuse. We retrospectively analyzed 110 urine patient samples (average age 36,3 $\pm 14,7$, age range 15-81 years old) coming from ER (39), from Ser.T. (61) and blood transfusion department (10) of Ospedali Riuniti di Ancona, Italy. Blood trasfusion department samples were employed as negative control. Samples were assayed on CDx90 analyzer using immunoturbidimetric ARK Pregabalin Urine Assay (Tema Ricerca Srl, Italy). Application was elaborated in collaboration with Tema Ricerca. Positive samples were confirmed with chromatographic method (HPLC).78 patients (71\%) were regular user of benzodiazepines, 15 of them (19\%) have been found positive for PRG, also confirmed by HPLC. 11 PRG positive patients (73,3\%) were found with very high level of PRG (>2000 ng/ml). These patients showed positivity even for other drugs of abuse such as cannabinoids, opiates, cocaine, amphetamines or alcohol. Our preliminary study confirms that PRG abuse is taking place and the potential hazard of this drug should not be underrated. Misuse of this therapeutic drug represents a serious emerging problem that must be monitored carefully through a rapid and reliable assay, now possible by automatic analyzers. 


\title{
EMERGING DRUGS ADDICTION: NIR SPECTROSCOPY AND CHEMOMETRICS FOR THE EARLY DETECTION OF NEW PSYCHOACTIVE SUBSTANCES
}

\author{
R. Risoluti', A. Gregori², L. Ripani², S. Materazzi ${ }^{1}$ \\ ${ }^{1}$ Dipartimento di Chimica, Università “Sapienza”, Roma \\ ${ }^{2}$ Reparto Investigazioni Scientifiche (RIS), Arma dei Carabinieri, Roma
}

\begin{abstract}
Near infrared spectroscopy (NIRs) proved to be a fast and non destructive tool for the detection of different compounds in forensic matrices [1]. This study investigated the feasibility of using NIR coupled to chemometrics calibration to detect new psychoactive substances in street samples. The capabilities of this approach in forensic chemistry were assessed in the determination of new molecules appeared in the illicit market and often claimed to contain "non-illegal" compounds, although exhibiting important psychoactive effects. The study focused on synthetic molecules belonging to the classes of synthetic cannabinoids, phenethylamines and cathinones. For each sample, NIR spectra in reflectance mode were acquired and chemometric tools based on Principal Component Analysis (PCA) were used to built a multivariate model of prediction for the screening of new emerging substances. The approach was validated comparing results with officials methods and successfully applied for "in site" determination of illicit drugs in confiscated samples, in cooperation with the Italian Scientific Investigation Department (Carabinieri RIS) of Rome. The achieved results allow to consider NIR spectroscopy analysis followed by chemometrics as a fast, cost-effective and useful tool for the preliminary determination of new psychoactive substances in forensic science.
\end{abstract}

1. Risoluti R, Materazzi S, Gregori A, Ripani L. Early detection of emerging street drugs by near infrared spectroscopy and chemometrics. Talanta 2016;153:407-13. DOI:10.1016/j.talanta.2016.02.044.

\section{THE DIFFUSION, STANDARDIZATION AND HARMONIZATION OF CEREBROSPINAL FLUID BIO- MARKERS ANALYSIS FOR THE DIAGNOSIS OF ALZHEIMER'S DISEASE: THE ITALIAN SELFIE}

\author{
G. Sancesario ${ }^{1}$, S. Toniolo ${ }^{2}$, S. Di Santo ${ }^{2}$, D. Chiasserini ${ }^{3}$, M. Pieri ${ }^{6}$, L. Parnetti ${ }^{3}$, M. Musicco ${ }^{5}$, G. Bernardi ${ }^{4}$, S. Bernardini ${ }^{6}$, Study Group \\ of Clinical Biochemistry of Biological Fluids other than Blood - SIBioC, SINdem-ITALPLANED \\ ${ }^{1}$ Dept. of Clinical and Behavioural Neurology, Santa Lucia Foundation IRCCS, Rome \\ ${ }^{2}$ Dept. of System Medicine, Tor Vergata University, Rome \\ ${ }^{3}$ Dept of Medicine, Center for Memory Disturbances, University of Perugia, Perugia \\ ${ }^{4}$ IRCCS C. Besta Neurologic Foundation, Milan \\ ${ }^{5}$ Epidemiology and Biostatistics Unit, National Research Council, Milan \\ ${ }^{6}$ Dept. of Experimental Medicine, Tor Vergata University, Rome
}

\begin{abstract}
Although the use of cerebrospinal fluid (CSF) biomarkers, amyloid $\beta 1-42$, tau and phosphorylated tau, has diagnostic and prognostic values $^{1}$, its diffusion is still limited in clinical practice, and only a restricted number of patients receive an integrated clinico-biological diagnosis. By a survey, we aimed at doing a "selfie" of the use of CSF biomarkers in the clinical practice in Italy, to understand the diffusion of CSF analysis and the distribution of CSF laboratories. Moreover, we considered pre analytical procedures, the methods used, the cut offs and the participation to Quality Control programs. We conducted a nationwide survey in March-May 2016, using an online questionnaire, sent to the members of SIBioC, SINdem-ITALPLANED and to main Neurological Clinics all over Italy ( $\mathrm{n}=1815$ ). Anonymous data were collected and analyzed. Based on our "selfie" study, in Italy AD biomarker' CSF analysis is currently performed in 24 laboratories, which can be distinguished as "internal laboratories" performing analysis just for their own hospital (9/24), and "centralized laboratories" (15/24), which provide biomarkers analysis for their own and for a network of neighboring hospitals. Indeed, 15 hospitals lack the service of an internal laboratory and send CSF samples to centralized laboratories. The distribution of laboratory centers varies along the territory. Surprisingly, nine regions lack CSF laboratories. The number of analyses is generally less than ten or less than twenty per month (39.13 and $47.83 \%$ of the responses); only in a few laboratories it is more than 20 per month (13.04\%). In $70.59 \%$ of the laboratories there is a standardization of pre analytical procedures; only some laboratories (54.17\%) participate in International Quality Control Programs. Moreover, there is no harmonization of cut-offs for the three biomarkers among centers. In Italy, the use of CSF biomarkers in clinical practice is patchy. The aim would be to offer to a larger number of patients an early and more accurate diagnosis and therapy. A standardization of pre analytical procedures and harmonization of cut offs are needed.
\end{abstract}

Dubois B, et al. Lancet Neurol 2014;13:614-629. 


\title{
ANTI-AQUAPORIN-4 ANTIBODIES LONGITUDINAL TITRATION IN NMO PATIENTS TREATED WITH RITUXIMAB: INVOLVEMENT IN THE PATHOGENESIS OF THE DISEASE, RITUXIMAB IMPACT, AND CLINICAL PRACTICE
}

\author{
F. Marnetto, A. Sala, P. Valentino, L. Granieri, A. Bertolotto \\ Neurologia 2 - Centro Riferimento regionale Sclerosi Multipla (CReSM) \& Neuroscience Institute Cavalieri Ottolenghi, Osp. S. Luigi, \\ Orbassano (TO)
}

\begin{abstract}
Neuromyelitis optica (NMO) is a severe autoimmune disorder of the central nervous system with inflammatory lesions restricted to the optic nerves and spinal cord. NMO is associated with autoantibodies to the water channel aquaporin-4 (AQP4). Chimeric monoclonal antibody Rituximab (RTX) have been shown to be effective in NMO; RTX reinfusion timing is based on monitoring the percentage of memory CD19+ B cells. Numerous studies analysed AQP4-Antibody (AQP4-Ab) titres, its correlation with the clinical course of disease, or during different treatments, to support a pathogenic role of these antibodies, with heterogeneous results. Our aims were to investigate the biological value of AQP4-Ab titer in the mechanism of disease and during RTX therapy, and to evaluate the usefulness of AQP4-Ab titration in the clinical practice. We determined AQP4-Ab titre in 322 serum samples from 7 NMO patients treated with RTX for a long follow-up (median 65 months), using an immunofluorescence cell based assay commercialized by Euroimmun. We found that 1) AQP4-Ab titres correlate with clinical disease activity with higher titres during and preceding clinical relapses compared with remission periods. 2) AQP4-Ab levels are reduced under RTX treatment; moreover, RTX induce a marked reduction in AQP4-Ab titres 3 months after infusion, as also during a 6 years follow-up. 3) AQP4-Ab levels were further analysed based on clinical outcomes (Annualized Relapse Rate and Expanded Disability Status Scale) evaluated for each patient to define treatment responsiveness: in the long term follow up, AQP4-Ab levels decrease in the majority of responder patients, while an increase in $\mathrm{Ab}$ titer occurs in the non responder one. This different trend is observed after the first two-years of therapy. 4) Correlation between every increase of CD 19+ B cells, AQP4-Ab titer and clinical relapses was also evaluated showing a low association. Results obtained demonstrate a good relationship between AQP4-Ab levels, clinical state and response to RTX therapy, supporting their involvement in the pathogenesis of NMO; in particular RTX reduce Abs levels in responder patients, providing information for the treatment efficacy after the first two years of therapy.
\end{abstract}

Granieri L, et al. Evaluation of multiparametric immunofluorescence assay for standardization of neuromyelitis optica serology. Plos One $2012 ; 7(6)$.

\section{THE USE OF GLYCATED ALBUMIN IN THE MANAGEMENT OF DIABETES MELLITUS}

\author{
E. Dozio \\ Milano
}

Glycated albumin (GA) is considered useful for assessing the degree of protein glycation directly dependent on glucose exposure. The conventional biomarkers employed for screening and monitoring diabetes mellitus (DM) include fasting glucose, postprandial glucose and glycated hemoglobin (HbA1c). Although they provide useful information, in some clinical conditions they are inadequate. GA is an interesting biomarker for diabetes mellitus: 1) as it is an intermediate-term marker of the glycemic status, it gives more information than the short-term (glycemia) and long-term (HbA1c) biomarkers currently employed in clinical practice; 2) in specific clinical conditions (altered erythrocyte lifespan, pregnancy and end-stage renal disease) it should be preferred to HbA1c; 3) it is probably also useful for diabetes mellitus screening and risk stratification of diabetes-related complications. HbA1c may be affected by any condition affecting erythrocyte lifespan (hemolytic anemia, hemorrhage, folate and vitamin B12 deficiency anemia, aplastic anemia, nephropathy) and hemoglobin metabolism (variant hemoglobin, thalassemia). Unlike HbA1c, GA is not influenced by their lifespan and is also independent of iron deficiency. In pregnancy HbA1c suffers some limits as an indicator of glycemic control since it raises from the second to the third trimester probably due to iron deficiency. Unlike HbA1c, GA is not affected by iron deficiency and, as an intermediate-term glycemic marker (albumin turnover is shorter than erythrocyte lifespan - 20 vs. 120 days), it enables pregnant women with DM to maintain a stricter glycemic control, important to lower the risk of fetal and maternal complications. GA is also a useful biomarker for monitoring diabetes mellitus in newborns due to the high levels of fetal hemoglobin. Patients with diabetes and end-stage renal diseases under dialysis also cannot be efficiently managed with HbA1c, because of the reduced persistence of red blood cells due to mechanical disruption, lower hemoglobin and erythropoietin concentration. GA may be a better indicator of their glycemic status. GA may be a useful diagnostic tool for diabetes screening in the general population and in individuals with a pre-diabetic condition. GA also rises sooner than HbA1c when glycemic status worsens, probably due to albumin biochemistry and its half-life. This means that GA is more useful as an indicator of glycemic status in all those conditions requiring short-term control of changes in glycemia, such as after the start or modifications of diabetes treatments. GA may be also directly implicated in the development of different complications related to diabetes, playing a role as a pathogenic molecule. However, in some specific disorders GA levels are either lower or higher than the mean plasma glucose concentration, mainly because of changes in the albumin metabolism.

In conclusion, the introduction of this biomarker in clinical practice could help clinicians in the diagnosis and monitoring of diabetes mellitus and in planning measures to prevent long-term diabetes complications. 


\title{
PARAPROTEINEMIAS AND THE KIDNEY
}

\author{
G. Quattrocchio, D. Roccatello \\ Struttura Complessa a Direzione Universitaria di Nefrologia e Dialisi \& CMID, Ospedale G. Bosco e Università di Torino
}

The finding of a monoclonal gammopathy (or paraproteinemia) in the serum and/or urine of a patient suggests the existence of a clonal proliferation of B-lymphocytes or plasma cells, and could represent either an overt malignant disease such as multiple myeloma (MM) and Waldenström macroglobulinemia or a premalignant condition called monoclonal gammopathy of undetermined significance (MGUS). Monoclonal proteins are usually detected by protein electrophoresis (PEP) combined with immunofixation electrophoresis (IFE) of the serum and/ or of a sample of urine. However, sometimes it can be very challenging to detect serum or urinary monoclonal immunoglobulins, particularly in cases of isolated immunoglobulin light chain disorders. Recently, serum immunoglobulin free light chain (FLC) assays with accurate quantification of both light chain isotypes and automatic calculation of the k/lambda FLC ratio have been introduced in the laboratory armamentarium leading to major improvements in early diagnosis and management of patients with paraproteinemias.

The kidney is frequently involved in monoclonal gammopathies and a wide variety of histopathological lesions with different clinical presentations can be encountered, depending on the amount and type of the circulating paraprotein (intact immunoglobulin or fragments like heavy and light chains). The term monoclonal gammopathy of renal significance (MGRS) has recently been introduced, and encompasses renal disorders caused by a monoclonal immunoglobulin secreted by a nonmalignant B-cell clone generally consistent with MGUS. Kidney biopsy is warranted to identify the exact nature of the lesion and to evaluate the severity of renal disease in order to define an adequate treatment. We present the clinical picture, the complex diagnostic workup and the individual therapeutic choices of three typical patients with three different types of paraprotein-related kidney diseases: 1) Cast nephropathy, 2) Light chain-associated (AL) amyloidosis, and 3) Monoclonal Ig deposition disease.

Serum and urinary PEP, IFE and FLC assays are indispensable to detect monoclonal paraproteins. Bone marrow aspirate and biopsy show the percentage of bone marrow plasma cells allowing the differentiation between MGUS, smoldering MM and overt MM. Kidney biopsy is crucial to identify the type and extension or renal lesions. The treatment is based on different chemotherapeutic regimens, which in some situations can be combined with removal of circulating paraproteins by means of extracorporeal therapies, such as plasma exchange, high cut-off hemodialysis and supra-hemodiafiltration with endogenous reinfusion. Interdisciplinary collaboration between hematologists and nephrologists is necessary, particularly in sharing a common therapeutic approach in some patients with MGRS.

\section{STANDARDIZATION OF FECAL CALPROTECTIN ANALYSIS}

\author{
A. Padoan \\ Department of Laboratory Medicine, University-Hospital of Padova, Italy
}

Fecal calprotectin (fCal) supports diagnosis and monitoring of inflammatory bowel diseases (IBDs). Different cut-offs are recommended for different clinical applications: $50 \mu \mathrm{g} / \mathrm{g}$ to rule-out IBDs, 150 to $300 \mu \mathrm{g} / \mathrm{g}$ to rule-in for IBDs relapse. A pre-analytical phase (sample weighting and dilution) precedes the analytical fCal measurement, which might be based on ELISA, chemiluminescent, RIA or turbidimetric assays, the overall variability of a single stool sample analysis depending on both the pre- and analytical phases. The intra-spot CV was estimated to be $25 \%$, while the overall biological variability $58 \%$, being this value even higher when among diseased patients $(1,2)$. Both analytical and biological variability might have a relevant impact on results interpretation, especially when values are close to the established cut-offs, thus limiting the efficacy of decision making strategies based on single test results. One of the main efforts of Clinical Laboratories should be oriented in reducing the analytical component of this variability by standardization and improvement in quality control by internal and EQA schemes. Currently, non-stool internal quality materials and EQA schemes with stool samples are used, but no standard material suitable for fecal sample analyses is available. In the absence of a standard reference material, a marker of accuracy is measurement uncertainty (MU). MU could be calculated by imprecision, trueness and bias uncertainty. By analysing 18 EQA results, we estimated fCal MU to be $23 \mu \mathrm{g} / \mathrm{g}$ and $175 \mu \mathrm{g} / \mathrm{g}$ for mean values below or above $200 \mu \mathrm{g} / \mathrm{g}$ respectively, confirming a concentration related MU. An added calprotectin analyte is now under evaluation for EQA schemes. We evaluated whether a recombinant standard (PRCA, DiaSorin Inc., USA) is suitable for fCal imprecision assessment by PhiCal Calprotectin ELISA (Immundiagnostik, Germany). MALDI-TOF/MS of PRCA confirmed its purity, showing only S100A8 and S100A9 peaks, which form the hetero-dimer Calprotectin. PRCA dissolved at the final concentration of 840, 420 and $210 \mathrm{ng} / \mu \mathrm{L}$ were diluted 1:25 in stool extraction buffer before ELISA, to reproduce the entire pre-analytical and analytical process. The expected results (336, $168 \mathrm{and} 84 \mathrm{ng} / \mathrm{mL}) \mathrm{chosen}$ were close to 300, 150 and $50 \mathrm{ug} / \mathrm{g}$ diagnostic cut-offs. Biases were: $-1.2 \%,-4 \%$ and $+6.6 \%$ from two replicated measures for the 336,168 and 84 $\mathrm{ng} / \mathrm{mL}$ targets respectively. Inter-assay imprecisions (11 independent assays) were: mean $=331.84 \mathrm{ng} / \mathrm{mL}, \mathrm{CV}=6 \%$; $\mathrm{mean}=175.7 \mathrm{ng} / \mathrm{mL}, \mathrm{CV}=9 \%$; mean=100.3, ng/mL, CV=21\%. In conclusion, a recombinant standard calprotectin might be useful as internal quality control for fCal assay.

\section{References}

1. Husebye E, Tøn H, Johne B. Biological variability of fecal calprotectin in patients referred for colonoscopy without colonic inflammation or neoplasm. Am J Gastroenterol 2001;96(9):2683-7.

2. Calafat M, Cabré E, Mañosa M, Lobatón T, Marín L, Domènech E. High within-day variability of fecal calprotectin levels in patients with active ulcerative colitis: what is the best timing for stool sampling? Inflamm Bowel Dis 2015;21(5):1072-6. 


\title{
A CASE OF HEMATOLOGICAL INVOLVEMENT IN CELIAC DISEASE
}

\author{
F. de Liso ${ }^{1}$, M. Ronchi ${ }^{1}$, C. Matinato ${ }^{1}$, N.N. Fantini' ${ }^{2}$, R. Maiavacca ${ }^{1}$ \\ ${ }^{1}$ Lab. Analisi Chimico Cliniche e Microbiologia, and ${ }^{2}$ Lab. Ematologia, Fondazione IRCCS Ca' Granda Ospedale Maggiore Policlinico, Milano
}

We report a case of a pregnant 39 year-old woman (22th week) with a condition of leucopenia/neutropenia (WBC 2.400/mmc; N 400/mmc), iron-deficiency anemia (RBC 3.620.000/mmc, Hb 9.4 g/dL, MCV $82.3 \mathrm{fL}$, serum ferritin $7 \mathrm{ng} / \mathrm{mL}$ ) and a slight elevation of transaminase (ALT $34 \mathrm{U} / \mathrm{L}$, AST $38 \mathrm{U} / \mathrm{L}$ ) whose causes were unclear, without family history of congenital anemia and leucopenia. The patient has been characterized by this hematological setting for ten years and medical management has been consisted by the treatment of symptoms (iron therapy). She arrived at our outpatient pregnancy clinic and the gynecologist required an hematological counseling. The hematologist decided to investigate the immunological status, assuming a possible autoimmune condition, which could justify the patient's long-term alterations. At first, anti-nuclear (ANA) and anti-double stranded DNA (anti-dsDNA) antibodies were required and were negative, but a fibrillar filamentous fluorescence of cytoplasm on HEp-2 cells (the gold standard substrate for detecting ANA) was observed. In order to better understand this fluorescence, the laboratory decided to evaluate anti-smooth muscle antibodies (ASMA) on rat Liver-Kidney-Stomach (LKS) section. Unexpectedly, a fluorescence reticulin-like was revealed and this result suggested to investigate the presence of celiac disease (CD). CD is an immune-mediated small intestinal enteropathy. Traditionally patients with CD have intestinal symptoms but over time the newly diagnosed patients can present with a wide range of symptoms and signs, including anemia and adverse pregnancy outcomes. According to guidelines, diagnosis of CD is by serology and duodenal biopsy (postponed until postpartum), and in this case we could perform only the serum panel for a new diagnosis of CD consisting in the assessment of total IgA (tIgA, to rule out a deficit of tIgA), IgA to tissue transglutaminase (TTG) and, if positive, IgA to endomisium (EMA). As the tests were strongly positive (TTG $>128 \mathrm{U} / \mathrm{mL}$, EMA positive), clinicians decided to start the gluten free diet with an improvement of WBC count $(6.100 / \mathrm{mmc})$, Hb levels $(11 \mathrm{~g} / \mathrm{dL})$, iron status (ferritin $21 \mathrm{ng} / \mathrm{mL}$ ) and transaminase values (ALT $18 \mathrm{U} / \mathrm{L}$, AST $21 \mathrm{U} / \mathrm{L})$ already after one month.

Ludvigsson JF, et al. Gut 2014;63:1210-1228.

\section{DYNAMIC CHANGE OF TnI IN PRESENCE OF ST SEGMENT ELEVATION BUT NO OBSTRUCTIVE CORONARY DISEASE}

\author{
C. Bellia ${ }^{1}$, B. Lo Sasso ${ }^{1}$, G. Bivona ${ }^{1}$, G. Novo ${ }^{2}$, M. Ciaccio ${ }^{1}$ \\ ${ }^{1}$ Sezione Biochimica Clinica e Medicina Molecolare, Dipartimento di Biopatologia e Biotecnologie Mediche, Università degli Studi di Palermo \\ ${ }^{2}$ Cattedra di Cardiologia, AOUP Policlinico P. Giaccone, Palermo
}

Introduction: the diagnosis of STEMI is based on the detection of new ST segment or T wave changes at ECG in presence of chest pain. Typically, a high increase of TnI is observed. Case description: The patient, a 66 years old woman, was admitted at the Emergency Department of Policlinico P. Giaccone, Palermo, for non radiating chest pain occurred in the last hours after an emotional stress. Her medical history comprised a positive familiar history for cardiovascular disease, arterial hypertension, gastroesophageal reflux disease, anxiety-depressive syndrome, dysthyroidism. On admission, blood pressure was 110/65 mmHg; ECG showed diffuse ST-T abnormalities with an elevation of ST segment; cardiac markers were TnI: 3.79 ng/ml and CPK: 374 U/L. After three hours TnI was $3.88 \mathrm{ng} / \mathrm{ml}$ and CPK was 377 U/L; after six hours cardiac markers concentrations declined (TnI: $2.88 \mathrm{ng} / \mathrm{ml}$; CPK: $339 \mathrm{U} / \mathrm{L}$ ) and became negative after 48 hours. Echocardiography showed apical akinesia and hyperkinesia of the basal segments of left ventricle (LV) with moderately impaired LV function (EF=43\%). No acute atherosclerotic lesions and no evidence of significant coronary artery stenosis were detected on angiography. Routine laboratory tests results were: AST: 33 U/L; ALT: 29 U/L; total cholesterol: 218 mg/dl; HDL cholesterol: 53 mg/dl; triglycerides: 88 mg/dl; glucose: 106 mg/dl; creatinine: 0.98 $\mathrm{mg} / \mathrm{dl}$; WBC: 9.76 10^3/microL; RBC: 4.49 10^6/microL; Hb: $12.1 \mathrm{~g} / \mathrm{dl}$; PLT: 310 10^3/microL. Given the moderate increase of TnI, the elevation of ST segment and the lack of stenosis or acute atherosclerotic lesions, the diagnosis of Takotsubo cardiomyopathy was suspected. Moreover, the Tn/EF ratio was 9, significantly lower than the cut-off of 60 proposed for differential diagnosis with AMI. Cardiac MRI ruled out both myocardial infarction and myocarditis and confirmed the diagnosis of Takotsubo cardiomyopathy. Supportive therapy with ACE-inhibitors, spironolactone and acetylsalicilic acid was initiated. After 4 days, echocardiography showed the complete recovery of LV function. Conclusion: a slight increase of $\mathrm{TnI}$ in presence of typical ST segment ECG abnormalities should be interpreted with caution, especially when it does not correlate with a significant but transient left ventricular dysfunction.

\section{A CASE OF VENTRICULAR WALL RUPTURE PREDICTED BY AN EXTREMELY INCREASED RDW VALUE}

\section{G. Lippi ${ }^{1}$, R. Aloe ${ }^{2}$, G. Cervellin ${ }^{3}$}

${ }^{1}$ Section of Clinical Biochemistry, University of Verona, Verona, Italy

${ }^{2}$ Laboratory of Clinical Chemistry and Hematology, Academic Hospital of Parma, Parma, Italy

${ }^{3}$ Emergency Department, Academic Hospital of Parma, Parma, Italy 
We describe the case of a 86-year old women, admitted to the emergency department (ED) of the University Hospital of Parma for acute dyspnea. The patient had a history of percutaneous transluminal coronary angioplasty (PTCA), chronic obstructive pulmonary disease (COPD) and osteoporosis, and was currently taking aspirin, clopidogrel, atorvastatin, phenytoin, lansoprazol, as well as calcium and vitamin D supplementation. At presentation she appeared dyspneic at rest, subcyanotic and with visible jugular vein distension. Immediately upon admission the patient underwent electrographic assessment, which was suggestive for a ST elevation myocardial infarction (STEMI) of the inferior wall and right ventricle, along with urgent laboratory testing. The main results of lab testing were a troponin I value of $54480 \mathrm{ng} / \mathrm{L}$ (reference range $[\mathrm{RR}]<60 \mathrm{ng} / \mathrm{L})$, hemoglobin $77 \mathrm{~g} / \mathrm{L}$ (RR 120-140 g/L), BNP $1885 \mathrm{pg} / \mathrm{mL}(\mathrm{RR}<150 \mathrm{pg} / \mathrm{mL}$ ) and estimated glomerular filtration rate (eGFR) $17 \mathrm{ml} / \mathrm{min}$ (RR $>60 \mathrm{ml} / \mathrm{min}$ ). Notably, the value of red blood cell distribution width (RDW) was $19.8 \%$, thereby extremely increased compared to its RR $(<14.8 \%)$. Due to the clinical status, ECG findings, increased troponin concentration and very high RDW value, the patient was immediately transferred to catheterization lab, where she eventually died $45 \mathrm{~min}$ afterward for ventricular wall rupture and cardiogenic shock. Albeit many laboratory data were indeed over the respective RRs, none of these (except RDW) was really predictive of such a dramatic complication of STEMI. This conclusion is in accord with current evidence attesting that RDW is an independent predictor of adverse outcome after myocardial infarction, exhibiting a predictive accuracy that is often greater than that of other and more expensive biomarkers. Despite the unfavourable outcome, this case report should be seen as a paradigmatic example of how the association between clinical perception and simple laboratory tests may provide a valuable aid for the clinical decision making in the ED. We hence conclude that major focus should be directed toward underestimated laboratory parameters such as the RDW by the emergency physicians, wherein the presence of a high degree of anisocytosis may always be considered an important negative prognostic factor.

\title{
CSF KAPPA AND LAMBDA FREE LIGHT CHAINS FOR THE DIAGNOSIS OF MULTIPLE SCLEROSIS
}

\author{
D. Lopergolo, M.T. Dell'Abate, D. Scribano, C. Zuppi, T. De Michele \\ Laboratorio Analisi 1, Fondazione Policlinico Universitario A. Gemelli, Rome, Italy
}

As today, oligoclonal band (OCB) analysis by mean of isoelectric focusing is considered the Gold Standard technique for Multiple Sclerosis (MS) diagnosis. Nevertheless, the technique is cumbersome and in the evaluation of samples yielding dubious OCB responses (i.e. a single band), OCB analysis reveals interpretative limitations. Notably, in our laboratory experience about $15 \%$ of total OCB analysis during the last year revealed dubious responses. Recently, free light chains (FLCs) has been suggested as potential diagnostic value in the context of MS. Thus, the kappa and lambda FLCs assay for intrathecal FLC quantification has emerged as a promising tool for supporting MS diagnosis. Here we describe 2 patients who underwent FLCs quantification in cerebrospinal fluid (CSF) and serum using Freelite ${ }^{\mathrm{TM}}$ (The Binding Site Ltd, UK), a nephelometric assay. Case 1 A 43 years old man presented with visual impairment and left homonym hemianopia. Microbiological examinations on CSF and serum were negative. The CSF diagnostic revealed a negative IgG index, positive CSF/serum albumin ratio (6.1) and OCB analysis indicated a borderline aspect (a single supernumerary OCB). CSF and serum $\kappa$ and $\lambda$ FLCs were within the reference range while $\kappa F L C$ index was positive $(13,6)$. Visual evoked potentials and MRI were indicative of retrobulbar optic neuritis suggesting a diagnosis of clinically isolated syndrome (CIS). Case 2 A 55 years old woman arrived to the hospital emergency with visual impairment in left eye lasting one week and mild retro-orbital pain. The CSF diagnostic revealed a negative CSF/serum albumin, negative IgG index and OCB analysis indicated a borderline aspect (a single supernumerary OCB). Interestingly, CSF $\kappa$ and $\lambda$ FLCs were respectively 1.90 and $0.45 \mathrm{mg} / \mathrm{L}$ and $\kappa F L C$ index was positive $(56,3)$ while serum FLCs were within the reference range. Evoked potentials and MRI were suggestive for optic neuritis and also indicated brain parenchymal signal abnormalities consistent with MS diagnosis. Conclusions The FLCs CSF test may be a valid alternative to support MS diagnostic protocols especially in the event of dubious or unclear OCB results, in order to confirm or reject all suspicions. FLCs assay may be performed as initial screening upon sample request submission.

\section{FREE LIGHT MEASUREMENT IDENTIFIES RELAPSE AND PROMPTS TO RECONSIDER AMYLOID TYPING IN A PATIENT WITH AL AMYLOIDOSIS}

\author{
P. Milani ${ }^{1}$, M. Basset ${ }^{1}$, F. Russo ${ }^{1}$, M. Nuvolone ${ }^{1}$, F. Lavatelli' ${ }^{1}$ T. Bosoni ${ }^{2}$, L. Pirolini ${ }^{2}$, A. Foli ${ }^{1}$, R. Albertini' ${ }^{2}$, G. Palladini ${ }^{1}$, G. Merlini ${ }^{3}$ \\ ${ }^{1}$ Centro per lo Studio e la Cura delle Amiloidosi Sistemiche, Fondazione IRCCS Policlinico San Matteo, Dip. di Medicina Molecolare, \\ Università di Pavia, Pavia \\ ${ }^{2}$ Lab. Analisi Chimico Cliniche, Fondazione IRCCS Policlinico San Matteo, Pavia \\ ${ }^{3}$ Direzione Scientifica, Fondazione IRCCS Policlinico San Matteo, Pavia
}

The detection and quantification of amyloidogenic monoclonal light chains (LC) is necessary for the diagnosis and evaluation of response to therapy in AL amyloidosis (Palladini et al. JCO 2012). A 69 years old woman with nephrotic syndrome (proteinuria $5 \mathrm{~g} / 24 \mathrm{~h}$ ), macroglossia and perorbital purpura was evaluated in another center in December 2001. A monoclonal IgG $\lambda$ was detected in serum and the urine immunofixation was negative. Creatinine was $0.98 \mathrm{mg} / \mathrm{dL}$ (u.r.l. $1.17 \mathrm{mg} / \mathrm{dL}$ ) and echocardiography was normal. The abdominal fat aspiration was positive. The diagnosis of AL $(\lambda)$ amyloidosis with renal and soft tissue involvement was made. In April 2002, the patient was started on melphalan and dexamethasone, and, after 4 cycles, was evaluated at our center. We documented a negative serum and urine immunofixation, with 
reduced proteinuria ( $2 \mathrm{~g} / 24 \mathrm{~h}$ ). Treatment was continued until cycle 6, and complete remission was confirmed in June 2002. Free light chain (FLC) measurement became available at our center in June 2003. FLCs were measured by the Binding Site assay on a BNII Dade-Behring nephelometer. FLC ratio on July 2003 was normal until June 2006, when proteinuria increased ( $8 \mathrm{~g} / 24 \mathrm{~h})$, serum creatinine was $1.9 \mathrm{mg} / \mathrm{dL}$, serum and urine immunofixation was still negative, but elevated $\kappa$-FLC concentration was documented (102 $\mathrm{mg} / \mathrm{L}, \kappa / \lambda$ ratio 5.69$)$. Since progression occurred in the absence of $\lambda$ monoclonal protein, but $\kappa$-FLC increased, we reconsidered the initial diagnosis. Thus, we repeated the abdominal fat aspirate for amyloid typing by immune-electron microscopy, that revealed $\kappa$-LC deposits. On frozen serum stored at the first evaluation at our center $\kappa$-FLC was $19 \mathrm{mg} / \mathrm{L}$ ( $\kappa / \lambda$ ratio 1.16). The final diagnosis was AL- $\kappa$ amyloidosis. The disease had responded to first line therapy, as confirmed by FLC measurements on frozen sera. A relapse was documented with $\kappa$-FLC increase. Thus, we started second line therapy with bortezomib and dexamethasone and, after 8 cycles, a second complete remission was obtained. In this case the FLC allowed the identification of amyloidogenic LC, that were not visible at immunofixation, enabling the timely detection of relapse. The identification of the LC forming the deposits and follow-up including FLC measurement are vital in AL amyloidosis.

\title{
MONITORING RESPONSE ASSESSMENT DURING TREATMENT WITH ANTI-CD38 IN A PATIENT WITH LIGHT CHAIN MULTIPLE MYELOMA
}

\author{
G. Topini', A. Fiorini' ${ }^{2}$, V. Panichi' ${ }^{1}$ R. Buzi ${ }^{4}$, M.T. Muratore ${ }^{4}$, M. Montanaro ${ }^{2}$ \\ ${ }^{1}$ Lab. di Citofluorimetria, Osp. S. Anna, Ronciglione \\ ${ }^{2}$ UOC Ematoogia, Osp. S. Anna, Ronciglione \\ ${ }^{3}$ Lab. di Citofluorimetria, Osp. S. Anna, Ronciglione \\ ${ }^{4}$ Lab. Analisi Chimico-Cliniche, Osp. Belcolle, Viterbo \\ ${ }^{5}$ Lab. Analisi Chimico-Cliniche, Osp. Belcolle, Viterbo \\ ${ }^{6}$ UOC Ematologia, Osp. S. Anna, Ronciglione
}

Daratumumab (DARA) is a human CD38-directed monoclonal antibody indicated for the treatment of patients(pts) with Multiple Myeloma(MM) who have received at least 3 prior lines of therapy including a proteosome inhibitor(PI)and an immunomodulatory agent (IA) or who are double refractory to a PI and an IA. We treated a 61 yrs old woman affected with light chain (LC) K MM with DARA from 31st of March till now. The patient was diagnosed with LC K MM in sept 2008. At diagnosis bone marrow (BM) plasmacells (PC) showed this cytometry pattern:CD138+, CD38+,CD56-. BJ was >2 g/dl, she had anemia,not renal failure. She underwent autologous BM transplantation after PAD induction (Doxorubicine, Bortezomib, Dexametasone)and then in the following relapses she was treated with bortezomib, thalidomide, lenalidomide, pomalidomide, cyclophosphamide, melphalan (M). In Jan 2016 she had a futher disease progression with worsening of renal failure.BM aspiration showed 75\% PC. At this stage of progressive disease we mainly monitored disease by free light chain (FLC) assay (Binding Site) because BJ could be underestimated in presence of renal failure. KFLC assay was $1225 \mathrm{mg} / \mathrm{L}$ (3.30-19.40), $\lambda$ FLC $4.96 \mathrm{mg} / \mathrm{L}$ (5.70-26.30), ratio 246.98 (0.261.65). She started renal adjusted M without improvement. K FLC assay increased to $9929 \mathrm{mg} / \mathrm{L}, \lambda 1.71 \mathrm{mg} / \mathrm{L}$ and ratio increased to 5806 , BJ $\mathrm{K} 625 \mathrm{mg} / \mathrm{L}$, creatinine $7 \mathrm{mg} / \mathrm{dl}$. So we decided to start DARA $16 \mathrm{mg} / \mathrm{kg}$ body with weekly iv infusion for the first 8 weeks (wks), then every 2 wks till 24 weeks and then every 4 wks until disease progression including our patient in a compassionate-use programme. K-FLC assay was performed during therapy and showed an improvement: after 2 courses K FLC was $881 \mathrm{mg} / \mathrm{L}$, ratio 1276, after $5 \mathrm{~K}$-FLC was $103.16 \mathrm{mg} / \mathrm{L}$, ratio 87.42, after 6 K FLC 147.9 mg/L, ratio 30.89, after 8 K FLC 119.79 mg/L, ratio 19.41, after 10 K FLC was 90.92, ratio 8.89. BM aspiration after 9 courses revealed 4\% PC: CD138+,CD38-,CD56- with down regulation of CD38 by DARA. Actually patient is clinically stable with improvement in blood count; she started however dialysis in May 2016. Therefore assessment of FLC assay is very important in this subset of MM pts with LC disease and with renal failure for monitoring response during therapy. Furthermore, DARA monotherapy, showed encouraging efficay in heavily pretreated and refractory pts with MM like this one.

\section{BIOCHEMICAL AND GENETIC CHARACTERIZATION OF A CHOLESTEROL BIOSYNTHESIS DEFECT: A NEW CASE OF STEROL-C4-METHYL OXIDASE DEFECT IN A YOUNG ITALIAN MALE}

\author{
M.P. Lenza', C. Sica ${ }^{1}$, M. Gelzo ${ }^{1}$, E. Procopio ${ }^{4}$, A. Dello Russo' ${ }^{1}$, G. Frisso ${ }^{3}$, G. Corso ${ }^{2}$, F. Salvatore ${ }^{3}$ \\ ${ }^{1}$ Dip. Medicina Molecolare e Biotecnologie Mediche, Università degli Studi di Napoli Federico II \\ ${ }^{2}$ Dip. Medicina Clinica e Sperimentale, Università degli Studi di Foggia \\ ${ }^{3}$ CEINGE Biotecnologie Avanzate s.c. a r.l. \\ ${ }^{4}$ Sez. Malattie Metaboliche e Muscolari ereditaria A.O.U. Meyer
}

Cholesterol plays a pivotal role in cell membrane physiology and in the biosynthesis of steroids, bile acids, and vitamin D. Inborn defects of cholesterol biosynthesis are a group of metabolic disorders presenting with multiple congenital anomalies, growth delay, and psychomotor disabilities. Recently, a new defect of cholesterol biosynthesis, involving the sterol-C-4-methyl oxidase (SC4MOL) enzyme, has been described in four patients as an autosomal recessive disease due to the defect of demethylation of C4-methylsterols (1). Herein, we describe a new case of SC4MOL deficiency. His clinical history reported bilateral congenital cataracts at the age of 8 months; at 4 years old, he showed psychomotor 
development delay and learning disabilities; at the age of 15 years, he showed small stature, microcephaly, cerebellum hypoplasia, obesity, and behavioural disorder. Despite numerous clinical, biochemical, and genetic examinations, such as array-CGH, X-fragile test, mitochondrial DNA sequencing, amino acids and organic acids in plasma and urine, the diagnosis was missed until the age of 19 years. Based on these evidences, a cholesterol biosynthesis defect was suspected. Sterol analysis by GC-FID and GC-MS showed higher levels of C4-monomethyland C4-dimethylsterols in plasma and red blood cell membranes, suggesting a defect of SC4MOL enzyme. Sequencing of the SC4MOL gene showed that the patient is compound heterozygote for two mutations: c.731A $>\mathrm{G}$ (p.Y244C), a known mutation, which substitutes an amino acid within highly conserved metal-binding domain; c.605G>A (p.G202E), not previously described, occurring in a trans-membrane site of fatty acid hydroxylase region. It is absent in EXAC database and in 250 healthy Caucasian individuals. Bioinformatics analysis suggests that this substitution may have a pathogenic role. Both his parents are found heterozygous. Therefore, genetic result supports the diagnosis of SC4MOL deficiency. In conclusion, integration between plasma and red blood cell membranes sterol analysis and genetic analysis allows to reach the definitive diagnosis. In addition, genetic result allows to differentiate among overlapping phenotypes, and to establish the exact reproductive risk.

Reference

1. He et al. 2014, BBA 1841:331.

\title{
PROJECT ON QUALITY INDICATORS OF IFCC WG-LEPS
}

\author{
M. Plebani, L. Sciacovelli \\ Department of Laboratory Medicine, University-Hospital of Padova, Italy
}

According to the International Standard for medical laboratories accreditation, ISO 15189:2012, "The laboratory shall establish quality indicators to monitor and evaluate performance throughout critical aspects of pre-examination, examination and post examination processes" and "The process of monitoring quality indicators shall be planned, which includes establishing the objectives, methodology, interpretation, limits, action plan and duration of measurement”. Therefore, the establishment of quality indicators (QIs) covering the entire testing process should be considered "a must” for complying with the requirements. The laboratory should, therefore, establish and periodically review QIs to monitor and evaluate performance throughout critical aspects of pre-, intra- and post-analytical processes. In fact, there is a large consensus on the vulnerability of the extra-analytical phases and on the need to improve extra-analytical procedures and processes, the current debate is about the strategies to be adopted to establish performance specifications (PSs) and tools for reducing extra-analytical errors. Errors in extra-analytical phases decrease the value of laboratory information, increase unjustified laboratory costs and affect patient safety. The Working Group of the International Federations of Laboratory Medicine "Laboratory Errors and Patient Safety" has launched a project on a model of quality indicators (MQI), available at the website www.ifcc-mqi.com. The project aims at the harmonization of QIs use in Laboratory Medicine through the management of External Quality Assurance Program (EQAP), the organization of meetings and scientific sessions, and the publications of papers in scientific journals. The harmonization of QIs, in addition to support compliance with the ISO 15189 requirements, allows the identification of a reliable state-of-the-art, which is the first step in defining the improvement goals, and contributes to the reduction of the errors and the improvement of patient safety. All laboratories, at international levels, have been called to contribute to the success of the project and to participate in the EQAP through the use of a common MQI, collection of data, and reporting of statistical data. The data collected from several laboratories worldwide have provided valuable insight on the state-of-the-art, especially as they were obtained using a harmonized list of QIs and with a homogeneous reporting system. A criterion to establish PSs has been proposed for each indicator in order to make easy the interpretation of QIs results and identification of action priorities. The definition of three different performance goals allows laboratories to evaluate how they are placed in comparison with other laboratories and if improvement actions are possible: the lower percentiles $\left(25^{\text {th }}\right)$ represent the better performances; the higher percentiles $\left(75^{\text {th }}\right)$ the worst performance.

The use of PSs is very important, first and foremost, to guide improvement programs and, in addition, represents a benchmark allowing interlaboratory comparison of performances.

\section{RECOMMENDATIONS FOR IDENTIFICATION AND MANAGEMENT OF CRITICAL VALUES IN CLINICAL LABORATORIES}

\author{
G. Lippi \\ Section of Clinical Biochemistry, University of Verona, Verona, Italy
}

The critical values, which are also conventionally referred to as "panic" or "alarm" values, are meant to emphasize a test result associated with an immediate and tangible risk for patient health, thus requiring timely notification to the healthcare personnel in charge of the patients, who should promptly establish the most appropriate therapeutic interventions (1).

Although the implementation of critical values reporting is now universally recognized as a vital standard for the good laboratory practice and a critical element for the accreditation process according to the ISO 15189 standard, the process of identification, routine implementation, 
notification and clinical management of critical values remains challenging around the globe. Despite reiterated claims by the Joint Commission (National Patient Safety Goals 01.01.01), supranational accreditation bodies and the IFCC working Group on Patient Safety, several lines of evidence attest that the importance of critical values reporting is still underrecognized and underestimated worldwide, nor validated practices for management in the laboratory are widely implemented (2,3). However, the application of standardized and universally agreed procedures for management of critical values appears now unavoidable to achieve a worldwide harmonization. In this perspective, valuable information can be taken from the guidelines issued by the Italian Society of Clinical Biochemistry and Laboratory Medicine (SIBioC), the Italian Society of Laboratory Medicine (SIMeL), with the Italian Committee for Standardization of Laboratory and Haematological Methods (CISMEL), through the Intersocietary Study Group (GdS) on "Standardization of extra-analytical variability of laboratory results" (4). In fact, the document is aimed to provide consensus recommendations for selection, application and management of critical values in clinical laboratories. Indeed, the development and introduction into hospital information systems of innovative information technology will facilitate to develop effective policies of critical values management, aimed to ultimately increase patient safety.

References

1. Piva E, Lippi G, Plebani M. Notification of abnormal and critical values: the road ahead. Am J Med 2010;123:e19.

2. Lippi G, Giavarina D, Montagnana M, Luca Salvagno G, Cappelletti P, Plebani M, et al. National survey on critical values reporting in a cohort of Italian laboratories. Clin Chem Lab Med 2007;45:1411-3.

3. Plebani M, Piva E. Notification of critical values. Biochem Med 2010;20:173-8.

4. Lippi G, Caputo M, Banfi G, Buttarello M, Ceriotti F, Daves M et al. Recommendations for detection and management of critical values in clinical laboratories. Biochim Clin 2008;32(3):209-216.

\title{
CHECK-LIST IN LABORATORY MEDICINE: AN IMPORTANT TOOL TO IMPROVE THE PATIENT SAFETY
}

\author{
A. Aita ${ }^{1}$, R. Marin ${ }^{1}$, E. Piva ${ }^{2}$, L. Sciacovelli², M. Plebani ${ }^{1}$ \\ ${ }^{1}$ Department of Medicine, University of Padova, Italy \\ ${ }^{2}$ Department of Laboratory Medicine, University-Hospital, Padova, Italy
}

\begin{abstract}
Background. Poorly standardized activities, human factors, patient crowding could affect the blood collection process leading to the preanalytical errors that impact on patient safety. The introduction of Checklist (CL) in laboratory medicine, prerogative of the surgical setting, could be considered an important tool to avoid the recurrence of procedural errors, ensuring the patient safety. Aim. This work aims to describe results concerning the implementation of a Check list (CL) on blood collection procedures in Laboratory Medicine. Methods. The project, focused on 3 outpatients phlebotomy sites (SMa, SMb and PN), involved 25 physicians and 15 nurses for a month. It concerns: analysis of literature and operating procedures carried out; selection of check-points (CPs); evaluation of CL draft; staff training; a brief period of experimentation; problems of analysis; release of a final CL. Results. 5661 CL were filled on 9469 venepunctures (59.8\%). The percentage of CPs filled in SMa, SMb and PN were respectively: 100- patient identification and label-sample-identification matching; 77.8, 71.0 and 58.5-vein selection; 97.8, 96.7 and 98.4-needle selection; 83.0, 85.8 and 90.6-tourniquet application time; 99.0, 97.9 and 98.7-tubes filling; 98.4, 97.4 and 98.7-tubes mixing; 28.0, 33.02 and 11.2-temperature transport; 20.8, 29.3 and 1.3-time transport. Conclusions. The CL was very helpful for staff in training, but difficult to use during the patients crowding. The insufficient number of prepared CL copies and difficulties over communication concerning purpose and methodology affected the project results. In order to prevent errors, a CL has to: include only critical CPs, be shared by all staff, be an integral part of a quality management system. Today, the CL focused on blood collection, is used in daily practice as a support tool to prevent and reduce procedural errors, in order to ensure the patient safety.
\end{abstract}

Reference

Lippi G, Mattiuzzi C, Banfi G, Buttarello M, Caputo M, et al. Proposta di una “checklist” per il prelievo di sangue venoso. Biochim Clin 2013;37:312-317.

\section{MISIDENTIFICATION IN LABORATORY MEDICINE: RESULTS OF THE TUSCANY SURVEY - RISK MANAGEMENT STUDY GROUP SIBIOC AND SIE}

\author{
C. Scapellato ${ }^{1}$, F. Balboni², M. Quercioli ${ }^{3}$, P. Pezzati4, G. Toccafondi ${ }^{5}$, P. Casprini ${ }^{6}$, A. Aita ${ }^{7}$, R. Guerranti' ${ }^{1}$, M. Fiorni ${ }^{8}$, G. Fuzzi ${ }^{9}$, \\ R. Tartaglia ${ }^{5}$ \\ ${ }^{1}$ UOC Patologia Clinica, Azienda Ospedaliera Universitaria Senese \\ ${ }^{2}$ Laboratorio Analisi, Istituto Fiorentino Cura e Assistenza, Firenze \\ ${ }^{3}$ Centro Regionale di Riferimento per il Controllo di Qualità, Regione Toscana \\ ${ }^{4}$ Dip. dei Servizi Lab. Analisi, AOU Careggi, Firenze \\ ${ }^{5}$ Centro Gestione Rischio Clinico e Sicurezza del Paziente, Regione Toscana
}


${ }^{6}$ Dipartimento Medicina di Laboratorio, USL Toscana Centro

${ }^{7}$ Dipartimento di Medicina (DIMED), Università degli Studi di Padova

${ }^{8}$ UOC Medicina di Laboratorio, Livorno, Azienda USL Toscana Nord Ovest

${ }^{9}$ Ricerche Cliniche "Prof. Manfredo Fanfani”, Firenze

The Risk Management and Patient Safety study group SIBioC with the Italian Society of Ergonomics developed a survey directed to Tuscany clinical laboratories aimed to investigate the level of awareness on the misidentification issues. The underlining concept is that clinical laboratories can play a central role in disseminating safety culture. The survey was designed by a scientific board and was validated by three validators. The survey consists of 30 questions many related to quality procedures from the non-conformity management to proactive or reactive analysis following adverse events: the focus is on human factors. 21 Tuscany laboratories were and 18 answered (86\%). All the Tuscany region is represented. $72 \%$ of structures declared to have accomplished ISO 9001:2008-2015 certification and 28\% other accreditation typology. A structured preanalytical phase is present in the $67 \%$ of laboratories and it is working $24 \mathrm{~h} 24,7$ days 7 in $50 \%$ of structures. Less than half (46\%) declares to treat non-conformities with proactive actions (FMECA: 56\% and 11\% in another way) and to register corrective actions systematically. A misidentified sample is analysed after error correction in $73 \%$, not analysed in the $40 \%$ and every action is traced $80 \%$. The non-conformity is telephonically communicated $100 \%$. There is the wide spread use of delta-check $80 \%$ with the purpose to identify misidentification errors although in the vast majority (70\%) there is not a structured procedure. In the $70 \%$ of cases a warning note is added. When a misidentification occurs, the clinical risk management is informed (60\%) and in $87 \%$ of cases a clinical audit or M\&M take places, followed by implementation plan. The IFCC project proposed by WG-LEPS is known only by the $33 \%$ of Tuscany laboratories. The results of the survey show that in Tuscany the safety culture is well disseminated and that laboratory personnel are well aware of the importance of preanalytical issues; however, there is room for improvement especially in the application of proactive techniques.

1. Bellandi T, Albolino S, Tartaglia R, et al. Human Factors and Ergonomics in Patient Safety Management in Human Factors and Ergonomics in Health Care and Patient Safety, Second Edition Ed. Pascale Carayon Taylor\&Francis, New York 2011.

\title{
START UP: RESEARCH AND DEVELOPMENT IN LIFE SCIENCE
}

\author{
F. Cariati ${ }^{2,3}$, V. D’Argenio ${ }^{1,2,3}$, N. Di Paola ${ }^{4}$, R. Vona ${ }^{4}$, R. Tomaiuolo ${ }^{1,2,3}$ \\ ${ }^{1}$ Dipartimento di Medicina Molecolare e Biotecnologie Mediche, Università degli Studi di Napoli Federico II \\ ${ }^{2}$ CEINGE-Biotecnologie Avanzate scarl \\ ${ }^{3}$ KronosDNA srl \\ ${ }^{4}$ Dipartimento di Economia, Management, Istituzioni, Università degli Studi di Napoli Federico II
}

In healthcare, in order for something to be classed as an innovation, it has to produce a positive impact, and improve processes. The innovation delivery is able to intensify the innovation effects in terms of improved patient experience, improved quality and decreased costs.

Although the importance of academic research to long-term economic growth is generally recognized, however the Italian context of medical research has not yet expressed its full economic potential.

The life sciences place specific barriers to the ability to identify business opportunities and transforming research results into marketable products. In order to identify solutions that are able to create the ideal conditions to trigger and develop the academic entrepreneurship in the Life Sciences (LS), the accelerator project focuses on the academic entrepreneurship activation mechanisms in healthcare, and the acceleration tools for healthcare academic spin-off companies.

In particular, the entrepreneurial path of One4Two will be described; it refers to a diagnostic kit that allows to perform the screening for genetic and chromosomal disorders related to infertility of couples in a single analytical run, by using a cutting-edge genomics method.

One4Two arises from the idea of a women's team. The original team worked for many years in scientific translational research and is now aspiring for entrepreneurship. Subsequently, the team has been enriched by the management component.

In effect, the typical and value-added feature of a start-up is the multidisciplinary composition of its founder team. It includes the medical and entrepreneurially-vocated component and the managerial one.

Through the launch of multidisciplinary research and educational paths, the know-how gained from the startup will be transferred to other academic spin-offs in healthcare, thus promoting the conditions that improve the academic research outcomes thanks to the consolidation of the University driving role in the innovation ecosystem, the enhancement of patenting activities and the contribution to the local socioeconomic development due to the spin-off companies (the so called "third mission" of the Universities).

\section{FROM TRADITIONAL BIOMARKERS TO CIRCULATING MICRORNAS IN CANCER}

\author{
M. Montagnana \\ Sezione di Biochimica Clinica, Dipartimento di Neuroscienze, Biomedicina e Movimento, AOUI Verona
}

The National Academy of Clinical Biochemistry (NACB) defines tumor markers as "..surrogate indicators that increase or decrease the clinician's suspicion that future clinically important events, such as cancer onset, recurrence, or progression or patient death, will or will not 
happen, and/or that a specific treatment will decrease the risk of such events” (1). These molecules may be produced and released either by tumor cells or by host cells, and their presence may be detected in the serum or other biological fluids, behaving as an indicator of the tumor presence. Typically, an ideal tumor marker should be easy to measure and reproducible, a positive result should only occur in patients with malignancy and quantitative levels would correlate with stage and response to treatment. Unfortunately, no one of the currently available tumor markers meets this ideal.

Epigenetics, conventionally defined as heritable change in gene expression that is not attributable to alteration of the DNA sequence, represents a new avenue in cancer research. Reliable evidence is accumulating that epigenetic mechanisms may play a key role in cancer progression and as well as in the onset of chemotherapy resistance (2). Moreover, since blood can easily be collected through a minimally invasive procedure, and also provides the ideal substrate for miRNAs analysis, the assessment of non-coding RNAs (ncRNAs) has been proposed as a valuable perspective for early diagnosis of different cancers (3). However, the clinical transferability of such tests is uncertain due to preanalytical, analytical and post-analytical variables (4).

Ovarian and endometrial cancer represent the most frequent gynecological cancer in developed countries. In patients affected by this neoplasia, 5-years survival rate is elevated when the diagnosis is made at an early stage, but it dramatically decreases when the cancer is diagnosed at stage IV. Given the high mortality rate of patients diagnosed with advanced cancers, the goal of gynecologists is to make a timely diagnosis and establish an early surgical and/or chemotherapic treatment.

In the last years, our research group performed some studies aimed to evaluate the diagnostic performance of both traditional, as CA125 and Human Epididymis Protein 4 (HE4), and epigenetic biomarkers in ovarian and endometrial cancer.

References

1. Sturgeon CM, Duffy MJ, Stenman UH, Lilja H, Brünner N, Chan DW, et al. National Academy of Clinical Biochemistry, National Academy of Clinical Biochemistry laboratory medicine practice guidelines for use of tumor markers in testicular, prostate, colorectal, breast, and ovarian cancers. Clin Chem 2008;54:e11-79.

2. Sharma S, Kelly TK, Jones PA. Epigenetics in cancer. Carcinogenesis 2010;31:27-36.

3. Pichler M, Calin GA. MicroRNAs in cancer: from developmental genes in worms to their clinical application in patients. Br J Cancer 2015;113:569-73.

4. Marzi MJ, Montani F, Carletti RM, Dezi F, Dama E, Bonizzi G, et al. Optimization and Standardization of Circulating MicroRNA Detection for Clinical Application: The miR-Test Case. Clin Chem 2016;62:743-54.

\title{
NEURODEGENERATIVE DISEASES AND OLIGOMERS: THE ROLE OF CLINICAL BIOCHEMISTRY
}

\author{
G.M. Sancesario \\ Dept. Clinical and Behavioural Neurology, Santa Lucia Foundation, Roma
}

Neurodegenerative diseases that belong to the group of proteinopathies share a common pathogenic mechanism, that is the formation of extracellular and/or intracellular deposits of abnormal misfolded proteins. This is the case of Alzheimer's, Parkinson's, Lewy Bodies, Huntington's and prion diseases, among others, in which distinct protein aggregates composed by amyloid $\beta$-protein (A $\beta$ ), tau, prion protein (PrP), $\alpha$-synuclein ( $\alpha$-syn) and huntingtin can be found in the brain of patients and seems to cause these disorders. Although such aggregation-prone proteins have entirely different sequences, they have the common propensity of fold into $\beta$-sheet-rich structures that contain one or more shared conformational epitopes and trigger different mechanisms of neurotoxicity and cognitive or functional impairment.

Recent evidences suggest that the formation of insoluble aggregates is preceded by soluble aggregates or oligomers that are not only correlated to fibril formation, but are also neurotoxic compounds. These emerging concepts are exemplified by Alzheimer's disease (AD), in which amyloid - $\beta$ oligomers (o-A $\beta$ ) adversely affect synaptic structure and plasticity [1], and Parkinson's disease (PD), for which in vivo and in vitro studies suggest a toxicity of soluble $\alpha$-syn oligomers (o- $\alpha \mathrm{Syn}$ ) for nigral dopaminergic neurons. The proposed role of o-A $\beta$ and o- $\alpha$ Syn in the brain pathology of AD [2] and PD [3] led to an extensive search of their presence in the CSF because, unlike insoluble fibrils deposited in brain tissue, soluble oligomers can diffuse in the CSF and, if detected in sensitive assays, they could constitute a diagnostic tool to be included in Clinical Laboratory Medicine of neurodegenerative diseases.

Several methods have been recently developed in the attempt to analyze diffusible oligomers in the CSF, but results on the normal levels and range are controversial. In $\mathrm{AD}$, some studies reported increased levels of o-A $\beta$, whereas others showed largely overlapping or decreased level respect to control or different diseases. Similarly, CSF level of $\alpha$-syn was lower in patients with PD and dementia with Lewy bodies than in those with AD, while other researchers have found no differences. Moreover, a significant decrease of t- $\alpha$-syn and an increase of o- $\alpha$-syn levels were found in PD patients [4] by using a specific double-ELISA, whereas other methods with excellent specificity for recombinant in vitro oligomerized $\alpha$-syn and oligomers in brainstem lysates of human $\alpha$-syn transgenic mice failed to detect oligomeric $\alpha$-syn in CSF [5]. Probably, the large variability in the results using different techniques could be due to the heterogeneity of oligomers, the presumably very low concentrations and the high background of monomeric forms.

The research in the field of CSF oligomers in AD and PD seems to be a winding road. A large variability in the results and the lack of a reliable method for assay still hamper the use of CSF oligomers in Clinical Biochemistry as new biomarkers for neurodegenerative disease. A great effort is required to validate a reliable method of analysis and harmonization of results. 
1. Hardy et al. J Neurochem 2009;110(4):1129-34.

2. Walsh et al. Nature 2002;416(6880):535-9.

3. Kalia et al. Ann Neurol 2013;73(2):155-69.

4. Parnetti et al. Front Aging Neurosci 2014;6:53.

5. Bidinosti et al. J Biol Chem 2012;287(40):33691-705.

\title{
POSTPRANDIAL CHOLESTEROL METABOLISM: A CLUE TO THE IMPACT OF TM6SF2 rs58542926 VARIANT ON LIVER AND CARDIOVASCULAR DISEASE IN NAFLD?
}

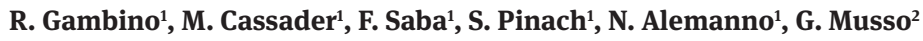 \\ ${ }^{1}$ Department of Internal Medicine, University of Turin, Italy \\ ${ }^{2}$ Gradenigo Hospital, Turin, Italy
}

Background: Nonalcoholic fatty liver disease (NAFLD) encompasses a histological spectrum, ranging from simple steatosis to steatosis plus necroinflammation (NASH), which can be differentiated only by liver biopsy. Genetic factors contribute to the pathogenesis of NAFLD. Genetic variation in the transmembrane 6 superfamily member 2 protein (TM6SF2) at rs58542926 was shown to confer susceptibility to NAFLD ${ }^{1}$. Objective: We examined the impact of this polymorphism on postprandial lipoprotein subfractions and on postprandial changes in cytokeratin-18 fragments in normolipidemic biopsy-proven NAFLD patients and matched healthy controls. Methods: fifty-five nonobese, nondiabetic, normolipidemic biopsy-proven NASH patients and 55 age, sex, BMI-matched healthy controls underwent an oral fat load test, with measurement of plasma triglyceride-rich lipoprotein subfractions, total cholesterol, oxidized LDL, non-esterified fatty acids, E-selectin, ICAM, and cytokeratin-18 fragments. Results: TM6SF2 T-allele carriers showed a lower postprandial triglyceridaemia and nefaemia, and a striking redistribution of cholesterol from smaller, more atherogenic VLDL2, LDL and oxLDL particles to larger VLDL1 subfractions than C-allele carriers. On multiple regression analysis, IAUC VLDL1-Cholesterol during the oral fat load independently predicted NAFLD activity score $(\beta=0.394, p=0.022)$, IAUC CK-18 ( $(=0.412, p=0.018)$ and fibrosis score $(\beta=0.402, p=0.019)$, while IAUC oxLDL predicted circulating E-selectin ( $\beta=0.418, p=0.011)$ and ICAM-1 ( $\beta=0.451, p=0.012$ ). Conclusions: These findings may partially explain the impact of TM6SF2 C $>$ T variant on liver injury and CVD risk in NAFLD. TM6SF2 C>T variant modulates dietary cholesterol fluxes, with T-allele diverting toxic cholesterol away from the vessel walls into the liver, thereby promoting liver injury. Consistently, circulating VLDL-Cholesterol closely correlated with hepatic cholesterol content, inflammation, fibrosis, and cell injury in NASH. If confirmed by larger follow-up studies, these findings may provide the rationale for evaluating approaches using cholesterol-lowering medications to alleviate hepatic cholesterol overload and liver injury in TM6SF2 T-allele carriers, irrespective of fasting blood cholesterol levels.

1. Zhou Y, et al. J Hepatol 2015;62:657-63.

\section{FULLY AUTOMATED MASSIVELY PARALLEL SEQUENCING (MPS) LIBRARY PREPARATION FOR GERMLINE BRCA1/2 MUTATION TESTING BY OMNIA LH100 AND LH75 (MASMEC) SYSTEMS}

\author{
M. De Bonis ${ }^{1}$, A. Costella ${ }^{1}$, V. Costantino ${ }^{2}$, A. Minucci ${ }^{1}$, E.D. Capoluongo ${ }^{1}$ \\ ${ }^{1}$ Laboratory of Clinical Molecular and Personalized Diagnostics, Institute of Biochemistry and Clinical Biochemistry, Catholic University, \\ Rome, Italy \\ ${ }^{2}$ R\&D Department Masmec Biomed-Masmec SPA Division, Modugno, Bari, Italy
}

Introduction. Library preparation for MPS is one of the most critical, on.bench and time-consuming steps in the MPS workflow. Automation of library preparation phase is the best option to avoid the risk of human-introduced error. Our objective was to report a rapid, automated solution to prepare Multiplicom-NGS based protocol for Illumina MiSeq ${ }^{\circledR}$ sequencer for BRCA1/2 genes and to analyze the performance of MPS in terms of number of sequences/run, coverage uniformity and number of variants detected. A total of 120 samples were used to evaluate the automated preparative process. Methods. Complete protocol for BRCA1/2 genes amplification by the BRCA MASTR Dx kit (Multiplicom, Niel, Belgium) was performed using OMNIA LH 100 and OMNIA LH 75 automated workstations designed and produced by MASMEC Biomed (Modugno, Bari). The LH 100 was equipped with a robot (X-Y-Z), 8 independent pipette channels and a layout with two racks for reagents and DNA samples, 9 deck positions for 96 well plates and different size tips and two heating-cooling units for controlled temperature steps. The LH 75 was prepared with a single pipette and a magnetic tool for analyzing 12 samples at the same time and 6 deck positions for 96 well plate and different size tips. Both workstations were controlled by MASMEC Framework software and were provided with UV lamp for decontamination to reduce the risk of cross-contamination. Results. OMNIA platforms were accurately customized to set up libraries preparation and purification process of 12 patients simultaneously per run in only 8-9 working hours (respect to 1-2 working days for manual execution). Because the workstations require very little hands-on time, 12 consecutive samples were prepared for the next automated amplification cycle while the first 12 samples were purified. Thanks to perfect setting of ratio between magnetic beads and DNA amount, a high-quality of BRCA1/2 libraries and MPS performance were obtained. Discussion. This study describes a new automated solution for fast and reproducible BRCA1/2 library 
preparation for MPS using a robotic workstation. Automated library preparation and MPS performance were comparable to a standard manual library preparation. The throughput of our pipeline was high positively improved by introducing these machines in our routine workflow.

\title{
IDENTIFICATION AND QUANTIFICATION OF URINARY MONOCLONAL PROTEINS BY CAPILLARY ELECTROPHORESIS IN AL AMYLOIDOSIS
}

\author{
P. Milani' ${ }^{1}$, G. Palladini ${ }^{1}$, F. Russo ${ }^{1}$, V. Valentini ${ }^{1}$, M. Basset ${ }^{1}$, T. Bosoni ${ }^{2}$, G. Ferraro ${ }^{1}$, L. Pirolini ${ }^{2}$, A. Foli ${ }^{1}$, F. Lavatelli ${ }^{1}$, F. Belvisi ${ }^{2}$, \\ M. Nuvolone ${ }^{1}$, R. Albertini ${ }^{2}$, G. Merlini ${ }^{3}$ \\ ${ }^{1}$ Centro per lo Studio e la Cura delle Amiloidosi Sistemiche, Fondazione IRCCS Policlinico San Matteo, Dip. di Medicina Molecolare, \\ Università di Pavia, Pavia \\ ${ }^{2}$ Lab. Analisi Chimico Cliniche, Fondazione IRCCS Policlinico San Matteo, Pavia \\ ${ }^{3}$ Direzione Scientifica, Fondazione IRCCS Policlinico San Matteo, Pavia
}

Identification and quantification of urinary monoclonal proteins (uMPs) is fundamental in the diagnosis and monitoring of monoclonal gammopathies (Kyle et al. Leukemia 2010). We prospectively assessed the performance of the Sebia Capillarys urine protein capillary electrophoresis (UPCE) and immunotyping in 75 patients with AL amyloidosis. Samples were tested with: homemade high-resolution agarose gel immunofixation electrophoresis (hr-IFE) of serum and concentrated (10 times) urine; commercial semi-automated agarose gel immunofixation of urine (Sebia Hydragel BJ on Hydrasys 2, SHBJ); UPCE and immunotyping (Sebia Capillarys 2 Flex Piercing Urine); quantification of circulating free light chains (FLC) by Freelite and N latex FLC. Urinary MPs were quantified using Sebia Phoresis software tools. Sixty-eight patients in whom uMPs were detected by hr-IFE were included in the study. A uMP was detected by UPCE in 62 cases (91\%), and was quantifiable in 55 (81\%). The median uMP excretion was $130 \mathrm{mg} / 24 \mathrm{~h}$ (range 10-1610). Nine of the 12 patients with dFLC $<50 \mathrm{mg} / \mathrm{L}$ (Freelite) had a quantifiable uMP (median $90 \mathrm{mg} / 24 \mathrm{~h}$ ). The uMP was also quantifiable on SHBJ in 51 patients (75\%). There was a good correlation between measurements of uMP excretion on UPCE and SHBJ (Pearson's $r=0.87,95 \% \mathrm{CI}$ 0.78-0.92). So far, 16 patients with quantifiable uMP and dFLC (Freelite) $>50 \mathrm{mg} / \mathrm{L}$ were treated and had response data at 3 months. Five subjects responded with a median $69 \%$ dFLC decrease (range 51-90\%). In all of them uMP excretion decreased (median 100\%, range 30-100\%). Among non-responders, only one had a relevant reduction in uMP (from 740 to $250 \mathrm{mg} / 24 \mathrm{~h}$, dFLC from 746 to $619 \mathrm{mg} / \mathrm{L}$ ) with stable renal function. Post-treatment UPCE was also available in 5 cases with baseline dFLC (Freelite) $<50 \mathrm{mg} / \mathrm{L}$. In 2 of them the uMP was still visible but was no longer quantifiable, in 2 it remained stable and in one uMP increased from 20 to $40 \mathrm{mg} / 24 \mathrm{~h}$. UPCE can identify uMPs in patients with AL amyloidosis with a good sensitivity, and can quantify uMP excretion as low as $10 \mathrm{mg} / 24 \mathrm{~h}$. Changes in uMP excretion can be monitored during therapy, including patients with low FLC disease. Further studies are warranted to evaluate the response assessment.

\section{ASSESSMENT OF IMMUNONEPHELOMETRY AS SCREENING METHOD FOR LABORATORY DIAGNOSIS OF BENCE JONES PROTEIN}

\author{
P. Natali, M. Varani, G. Patelli, S. Tagliavini, D. Carra, T. Trenti \\ S.C. Medicina di Laboratorio NOCSAE, Modena
}

Introduction - Free light chains $\kappa$ and $\lambda$ (FLC) monoclonal immunoglobulin in serum and urine (BJP) are markers of B-cell proliferative disorders. Immunofixation electrophoresis agarose gel in urine (UIFE) is the reference method for the determination of BJP, while the Immunonephelometric assay (INA) to detect FLC in urine is accepted as an initial screening method to exclude negative samples. The aim of the present study was to compare the performance of INA and UIFE methods in urine samples and to estimate the capability of INA to select BJP negative samples. Methods - The urine samples were received by Corelab NOCSAE Modena. One thousand of unconcentrated ones were analyzed by INA method, with FLC antiserum (New Scientific Company -NSC) by Nephelometer Beckman Coulter: we considered negative the urine samples with both the concentration of FLC $\mathrm{k}$ or $\lambda \leq 10 \mathrm{mg} / \mathrm{L}$, as suggested by NSC, and FLC $\mathrm{k}$ or $\lambda \leq 5 \mathrm{mg} / \mathrm{L}$, as indicated in the literature. Within 48 hours the same 1000 urine samples were tested by uIFE, with antiserum anti-heavy chains, anti-FLC k and $\lambda$ (Sebia): those that showed a homogeneous band in the FLC $\mathrm{k}$ or $\lambda$ track were considered as positive. The findings of both INA FLC $>10 \mathrm{mg} / \mathrm{L}$ and FLC $>5 \mathrm{mg} / \mathrm{L}$ (INA+) with uIFE results were compared. Results - Seventy-one urine were found positive for BJP (7.1\%). Data obtained with cut off FLC $\mathrm{k}$ or $\lambda$ $>10$ mg/L: urine 39 uIFE+ and INA+; 815 UIFE- and INA-; 114 UIFE - and INA+; 32 uIFE+ and INA-. Sensitivity 55\%; Specificity 88\%; LR + 4.5; LR- 0.5; Accuracy 85\%; Choen's kappa 0.3. Data obtained cut off FLC $\mathrm{k}$ or $\lambda>5 \mathrm{mg} / \mathrm{L}$ : urine 53 uIFE+ and INA+; 693 uIFE- and INA-; 236 uIFE - and INA+; 18 uIFE+ and INA-. Sensitivity 75\%; Specificity 75\%; LR+ 2.9; LR- 0.3; Accuracy 75\%; Choen's kappa 0.2. Conclusions - The results obtained using INA to select the negative urine for BJP are unsatisfactory considering both proposed cut off of FLC $\leq 10 \mathrm{mg} / \mathrm{L}$ and FLC $\leq 5$ $\mathrm{mg} / \mathrm{L}$. The correlation between the results of INA and UIFE methods is poor, as a high number of false positives were found and an even higher number of false negatives. Therefore INA is an unsuitable screening method even only to detect negative samples.

References

Graziani MS, et al. Guidelines for the analysis of Bence Jones protein. Clin Chem Lab Med 2003;41:338-46. 